Linköping Studies in Science and Technology

Dissertation No. 1870

\title{
Metamodel-Based Multidisciplinary Design Optimization of Automotive Structures
}

\author{
Ann-Britt Ryberg
}

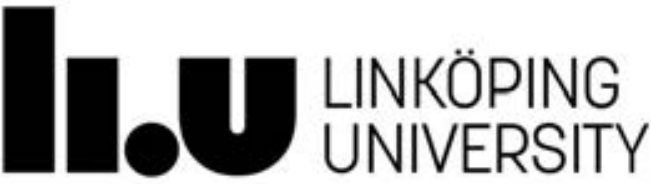

Division of Solid Mechanics

Department of Management and Engineering

Linköping University

SE-581 83 Linköping, Sweden

Linköping, October 2017 
Cover:

Illustration of a continuous metamodel, developed with the estimated guiding samples approach, representing a discontinuous response in two variables.

Printed by:

LiU-Tryck, Linköping, Sweden, 2017

ISBN 978-91-7685-482-2

ISSN 0345-7524

Distributed by:

Linköping University

Department of Management and Engineering

SE-581 83 Linköping, Sweden

Copyright (C) 2017 Ann-Britt Ryberg unless otherwise noted

No part of this publication may be reproduced, stored in a retrieval system, or be transmitted, in any form or by any means, electronic, mechanical, photocopying, recording, or otherwise, without prior permission of the author. 


\section{Preface}

The work presented in this thesis has been carried out within a $\mathrm{PhD}$ programme at the Division of Solid Mechanics, Linköping University. Financial support has been provided by the Swedish government agency for innovation Vinnova/FFI, and it has also been a part of the SFI/ProViking project ProOpt.

Some years ago when I began this journey, which I now approach the end of, I could never imagine how different some things would be today. I have learned that research is hard to plan. Setbacks, as rejection of articles, as well as happy events, as the birth of my children, have all made the journey longer than anticipated. I started my research studies as an employee of the car manufacturer Saab Automobile AB. After having spent many years working with different types of safety simulations, the intention was to find suitable methods for multidisciplinary design optimization that I could implement into the development process of the company. However, the financial situation for Saab Automobile $\mathrm{AB}$ became untenable and the company went into bankruptcy. I am therefore very grateful to my new employer Combitech AB that allowed me to continue my endeavours. Since Combitech $\mathrm{AB}$ is a technical consulting company, my focus has shifted towards improved methods rather than implementation during the latter part of the project. I am now eager to put my newly acquired knowledge into practice for our customers.

A number of people have been important for the outcome of the work presented in this thesis. First and foremost, I would like to express my deepest appreciation to my supervisor Professor Larsgunnar Nilsson for his guidance and support during the course of the work. Many thanks also go to my PhD student colleague Rebecka Domeij Bäckryd for our close collaboration and very fruitful discussions during the first years of the work. Additionally, my manager Tomas Sjödin deserves special appreciation for being one of the initiators of the project and for always supporting me. I am also grateful to all my colleagues, friends, and family for their support and interest in my work.

Finally, I would like to especially thank my beloved Henrik and our two lovely children Elsa and Gustav for making my life so enjoyable. I hope you will understand what is in this thesis some day and feel proud of me.

Ann-Britt Ryberg, Trollhättan, October 2017 


\section{Abstract}

Multidisciplinary design optimization (MDO) can be used in computer aided engineering (CAE) to efficiently improve and balance performance of automotive structures. However, large-scale MDO is not yet generally integrated within automotive product development due to several challenges, of which excessive computing times is the most important one. In this thesis, a metamodel-based MDO process that fits normal company organizations and CAE-based development processes is presented. The introduction of global metamodels offers means to increase computational efficiency and distribute work without implementing complicated multi-level MDO methods.

The presented MDO process is proven to be efficient for thickness optimization studies with the objective to minimize mass. It can also be used for spot weld optimization if the models are prepared correctly. A comparison of different methods reveals that topology optimization, which requires less model preparation and computational effort, is an alternative if load cases involving simulations of linear systems are judged to be of major importance.

A technical challenge when performing metamodel-based design optimization is lack of accuracy for metamodels representing complex responses including discontinuities, which are common in for example crashworthiness applications. The decision boundary from a support vector machine (SVM) can be used to identify the border between different types of deformation behaviour. In this thesis, this information is used to improve the accuracy of feedforward neural network metamodels. Three different approaches are tested; to split the design space and fit separate metamodels for the different regions, to add estimated guiding samples to the fitting set along the boundary before a global metamodel is fitted, and to use a special SVM-based sequential sampling method. Substantial improvements in accuracy are observed, and it is found that implementing SVM-based sequential sampling and estimated guiding samples can result in successful optimization studies for cases where more conventional methods fail.

Keywords: multidisciplinary design optimization (MDO); metamodel; artificial neural network (ANN); support vector machine (SVM); sequential sampling; crashworthiness; automotive structure; spot weld optimization 


\section{Populärvetenskaplig sammanfattning}

Multidisciplinär optimering (MDO) kan användas inom datorstödd produktutveckling för att förbättra och balansera fordonsstrukturers egenskaper på ett effektivt sätt. Idag är dock inte storskalig MDO generellt implementerad i fordonsindustrins produktutvecklingsprocess. Detta beror till stor del på de långa beräkningstider som krävs. I den här avhandlingen presenteras en process för att genomföra MDO som passar bilindustrins normala organisation och datorstödda utvecklingsprocess. Genom att införa globala metamodeller som approximerar de detaljerade simuleringsmodellernas responser erhålls både ökad effektivitet och möjlighet att låta involverade grupper arbeta självständigt och parallellt.

Den presenterade MDO-processen har visat sig effektiv för den vanligt förekommande uppgiften att optimera tjocklekarna $\mathrm{i}$ en tunnplåtskonstruktion med målet att minimera dess massa. Den kan också användas för att optimera antalet punktsvetsar i en konstruktion under förutsättning att modellerna byggs på rätt sätt. En jämförelse av olika metoder visar att topologioptimering, som kräver mindre förberedelser och datorresurser, kan vara ett alternativ i vissa fall.

En utmaning vid optimering med metamodeller är bristande noggrannhet hos metamodellerna när de skall representera vissa typer av komplexa responser. Sådana responser är vanliga vid t.ex. kollisionsförlopp där diskontinuiteter och olika deformationsbeteende kan erhållas beroende på de studerade designvariablernas värden. En stödvektormaskin (SVM) är en typ av maskininlärningsmetod som kan användas för att klassificera data och exempelvis identifiera gränsen mellan olika deformationsbeteenden. I den här avhandlingen används denna gräns för att på olika sätt öka metamodellers noggrannhet. Det visar sig att de föreslagna metoderna i många fall leder till bättre metamodeller och att de kan resultera i framgångsrika optimeringsstudier för fall där mer konventionella metoder inte fungerar. 


\section{List of Papers}

In this thesis, the following papers have been appended:

I. Bäckryd, R. D., Ryberg, A.-B., Nilsson, L. (2017). Multidisciplinary design optimisation methods for automotive structures. International Journal of Automotive and Mechanical Engineering 14(1):4050-4067. doi:10.15282/ijame.14.1.2017.17.0327

II. Ryberg, A.-B., Bäckryd, R. D., Nilsson, L. (2015). A metamodel-based multidisciplinary design optimization process for automotive structures. Engineering with Computers 31(4):711-728. doi:10.1007/s00366-014-0381-y

III. Ryberg, A.-B., Nilsson, L. (2016). Spot weld reduction methods for automotive structures. Structural and Multidisciplinary Optimization 53(4):923-934. doi:10.1007/s00158-015-1355-4

IV. Ryberg, A.-B., Nilsson, L. (2017). Improving metamodel accuracy for structural responses including discontinuities. Accepted.

V. Ryberg, A.-B., Nilsson, L. (2017). Improving accuracy of feedforward neural network metamodels for crashworthiness optimization. Submitted.

\section{Own contribution}

The work resulting in the two first papers have been a joint effort by Rebecka Domeij Bäckryd and me. My contribution to the first paper includes being an active partner during the writing process. As for the second paper, I have borne the main responsibility for planning and conducting the application example, as well as writing the paper. For the last three papers, I have been the main contributor in developing the ideas, performing the studies, and writing the papers. 
Other relevant publications by the author:

VI. Ryberg, A.-B., Bäckryd, R. D., Nilsson, L. (2012). Metamodel-based multidisciplinary design optimization for automotive applications. Technical report LIU-IEI-R-12/003. Division of Solid Mechanics, Linköping University.

http://urn.kb.se/resolve?urn=urn:nbn:se:liu:diva-84701

VII. Ryberg, A.-B. (2013). Metamodel-based design optimization - A multidisciplinary approach for automotive structures. Licentiate thesis No. 1565. Division of Solid Mechanics, Linköping University.

http://urn.kb.se/resolve?urn=urn:nbn:se:liu:diva-88136

(The printed licentiate thesis includes pre-print versions of Paper I and Paper II). 


\section{Contents}

Preface iii

$\begin{array}{lll}\text { Abstract } & \text { v }\end{array}$

Populärvetenskaplig sammanfattning vii

List of Papers $\quad$ ix

Contents $\quad$ xi

Part I - Theory and Background 1

1 Introduction 3

1.1 Scope of Work . . . . . . . . . . . . . . . . . . 4

1.2 Outline . . . . . . . . . . . . . . . . . 4

2 Structural Optimization 5

2.1 Multi-Objective Optimization . . . . . . . . . . . . . . . 5

2.2 Optimization Under Uncertainties . . . . . . . . . . . . . . . . . 6

2.3 Multidisciplinary Design Optimization. . . . . . . . . . . . . . . . 6

2.4 Metamodel-Based Design Optimization . . . . . . . . . . . . . . . 7

2.5 Optimization Algorithms . . . . . . . . . . . . . . . . . . 8

2.6 Topology Optimization. . . . . . . . . . . . . . . . . . . 9

3 Metamodel-Based Design Optimization $\quad 11$

3.1 Design of Experiments . . . . . . . . . . . . . . . . . . . . 12

3.1.1 Latin Hypercube Designs . . . . . . . . . . . . . . . . . . . . . . 13

3.1.2 Distance-Based Designs . . . . . . . . . . . . . . . . . . . . . . . 13

3.1 .3 Sequential Sampling . . . . . . . . . . . . . . . . . . 15

3.2 Metamodels . . . . . . . . . . . . . . . . 15

3.2.1 Feedforward Neural Networks. . . . . . . . . . . . . . . . . 15 
3.2.2 Radial Basis Function Neural Networks. . . . . . . . . . . . . . . . . 17

3.3 Metamodel Validation . . . . . . . . . . . . . . . . . . 18

3.3.1 Error Measures . . . . . . . . . . . . . . . . . . . . 19

3.3 .2 Cross Validation. . . . . . . . . . . . . . . . . . . . . 20

3.3 .3 Generalized Cross Validation . . . . . . . . . . . . . . 20

4 Multidisciplinary Design Optimization $\quad 21$

4.1 Single-Level Methods . . . . . . . . . . . . . . . . . . . . 22

4.2 Multi-Level Methods. . . . . . . . . . . . . . . . . . . . . 22

4.3 An MDO Process for Automotive Structures . . . . . . . . . . . . . 25

5 Support Vector Machines $\quad 29$

5.1 SVM Decision Boundary . . . . . . . . . . . . . . . . . . . . . 29

5.2 SVMs to Improve Metamodel Accuracy . . . . . . . . . . . . . . . . . . . 32

6 Summary of Appended Papers $\quad 35$

7 Conclusions and Outlook

$\begin{array}{ll}\text { References } & 41\end{array}$

Part II - Appended Papers

Paper I

Multidisciplinary design optimisation methods for automotive structures . . . . . 51

\section{Paper II}

A metamodel-based multidisciplinary design optimization process for automotive structures . . . . . . . . . . . . . . . . . 71

Paper III

Spot weld reduction methods for automotive structures . . . . . . . . . . 91

\section{Paper IV}

Improving metamodel accuracy for structural responses including discontinuities. 105

Paper V

Improving accuracy of feedforward neural network metamodels for crashworthiness optimization. . . . . . . . . . . . . . . . 137 
Part I

Theory and Background 



\section{Introduction}

Automotive companies are exposed to strong competition and are forced to improve their methods and processes in order to develop vehicles with improved performance faster and at a lower cost. A special challenge during development of automotive structures is to handle the conflicting demands of decreased mass and increased crashworthiness, which stem from new requirements on emission and safety, respectively.

During the development of a car, certain groups are responsible for the design of a subsystem, e.g. the body, the interior, or the chassis, while others are responsible for a performance aspect, e.g. safety, aerodynamics, or noise, vibration, and harshness (NVH). To be successful, these experts must cooperate in an organized an efficient manner. During the development, initial concepts are gradually refined to form the final product. Traditionally, the goal has been to find a feasible design, i.e. a design that meets all requirements. However, by the use of modern development methods, more optimized designs can be obtained. One such method is multidisciplinary design optimization (MDO), which is an approach to optimize designs considering different disciplines simultaneously. To minimize the mass of a vehicle body considering NVH and crashworthiness is a kind of MDO that is documented in several articles, see e.g. Craig et al. (2002), Sobieszczanski-Sobieski et al. (2001), Yang et al. (2001), Kodiyalam et al. (2004), Hoppe et al. (2005), Duddeck (2008), Sheldon et al. (2011), and Rakowska et al. (2015).

The potential of MDO has thus been identified by the automotive industry. However, MDO studies are not yet generally integrated within the product development process due to several challenges. First, large-scale MDO studies require that experts from different groups work together. Second, simulation models of the same status must be available. Furthermore, MDO is computationally expensive. To reduce the amount of computational resources needed, approximations of the original detailed simulation models, called metamodels, are often used. However, the accuracy of these models can be a concern, especially when they represent complex systems with strongly non-linear behaviour as found in for example different crash scenarios. 


\subsection{Scope of Work}

The work presented in this thesis is part of the research project "Robust and multidisciplinary design optimization of automotive structures" (reference numbers 200900314 and 2014-01340) partially funded by Vinnova/FFI. The primary aim of the project is to find suitable methods for implementing large-scale robust and multidisciplinary design optimization of automotive structures within current product development. Methods for dealing with optimization under uncertainties are covered by Shetty (2017). This thesis focuses on topics associated with simultaneous optimization of aspects related to different disciplines or load cases.

First, different solution methods for MDO are identified and evaluated (Paper I). Based on the findings, a metamodel-based MDO process is proposed and illustrated by a thickness optimization with the objective to minimize mass (Paper II). Thereafter, different methods for reducing the number of spot welds in a structure are evaluated from a multidisciplinary point of view (Paper III). The accuracy of metamodels is identified as one of the key enablers for successful MDO studies following the proposed MDO process. For that reason, methods for improving metamodel accuracy for complex responses by the help of support vector machines are then studied (Paper IV and V).

\subsection{Outline}

This thesis is divided into two parts. Part I gives the theory and background to the five appended papers that are included in Part II. The first chapter of Part I, following this introduction, gives a short introduction to different concepts related to structural optimization. The next two chapters introduce metamodel-based design optimization and multidisciplinary design optimization in more detail. These three chapters are partly based on the author's licentiate thesis (Ryberg 2013). Chapter 5 gives the theoretical background of support vector machines and how it can be used to improve metamodel accuracy. The five appended papers are summarized in Chapter 6 before the first part of the thesis is ended in Chapter 7 by summarizing the findings. 


\section{Structural Optimization}

A structure is collection of physical components that are arranged to carry loads and optimization is the process of finding the best solution to a problem. Hence, the task in structural optimization is to select suitable values for the design variables so that, under given constraints, the best possible performance of the structure is obtained. Mathematically this can be given as

$$
\begin{array}{cl}
\min _{\mathbf{x}} & f(\mathbf{x}) \\
\text { s.t. } & \mathbf{g}(\mathbf{x}) \leq \mathbf{0} \\
& \mathbf{x}^{\text {lower }} \leq \mathbf{x} \leq \mathbf{x}^{\text {upper }}
\end{array}
$$

where $f(\mathbf{x})$ is the objective function and the elements of the vector $\mathbf{g}(\mathbf{x})$ are the constraint functions. The elements of the vector $\mathbf{x}$ are the design variables that can vary within certain limits, called the design space. One or several analyzers are used to identify the values of the objective and constraint functions during the solution process. For structural optimization problems, the analyzers are often detailed finite element (FE) models. For a vector of design variables, an analyzer returns a number of responses, e.g. intrusions, energies, and mass. These responses are used to determine the values of the objective and constraints for that specific vector of design variables.

Three types of structural optimization problems are usually distinguished: size, shape, and topology optimization. In size optimization, the design variables represent structural properties, e.g. sheet thicknesses. In shape optimization, the design variables instead represent the shape of material boundaries. Topology optimization is the most general form of structural optimization and is used to find where material should be placed to be most effective.

\subsection{Multi-Objective Optimization}

The optimization problem defined in Equation (2.1) is a single-objective optimization problem. If the optimization involves more than one objective, it is termed multi-objective optimization. The simplest approach to solve such a problem is to convert it into a singleobjective problem, either by selecting one of the objective functions as the objective and 
treating the others as constraints, or by creating a new objective function as a weighted combination of the original objectives.

If a multi-objective optimization problem has conflicting objectives, there exist multiple optimal solutions to the problem. This set of solutions is termed Pareto optimal and are the solutions for which no other feasible solution yields a lower value of any objective without increasing the value of at least one other objective. For this type of problem, the goal is often to find a suitable trade-off between the conflicting objectives.

\subsection{Optimization Under Uncertainties}

It can be of vital importance to take uncertainties into account when a product is designed. An optimum design obtained through deterministic optimization as in Equation (2.1), i.e. without considering uncertainties, might become unreliable or non-robust when exposed to real world variations in the design variables or other parameters.

Methods dealing with optimization under uncertainties are often divided into two main categories. The first one is reliability-based design optimization (RBDO), where the goal is to find an optimum design for which the probability of failure is low. The second one is robust design optimization (RDO), a multi-objective problem where a design that performs well and is insensitive to variations is sought. However, a combination in which both the probability of failure is controlled, as in RBDO, and the spread of critical responses are reduced, as in $\mathrm{RDO}$, is also possible.

Methods that take uncertainties into account are not discussed further in this thesis. Still, the approaches developed can be used also in such a context.

\subsection{Multidisciplinary Design Optimization}

Multidisciplinary design optimization (MDO) is used to optimize a design taking into account more than one discipline simultaneously, i.e. the objective, constraints, or variables are related to different disciplines. Giesing and Barthelemy (1998) provide the following definition of MDO: "A methodology for the design of complex engineering systems and subsystems that coherently exploits the synergism of mutually interacting phenomena." In general, an improved design can be found when the interactions between different aspects of a product are considered compared to when these aspects are treated as isolated entities. Methodologies for MDO can also be applied to different load cases within one single discipline. The problem is then not multidisciplinary, but the idea to find improved solutions by taking advantage of the interactions between different aspects still remains. 
A typical MDO problem for automotive structures is to minimize the mass subject to a number of performance constraints that originate from different disciplines. Other possibilities include finding the best compromise between conflicting disciplinary requirements. As an example, the appropriate thicknesses of selected parts can be sought, but the most suitable shape or material quality etc. can also be identified.

Many different MDO solution methods have been proposed for which the optimization problem is either solved in its original form, or reformulated to a set of coordinated smaller optimization problems. More details about MDO are given in Chapter 4.

\subsection{Metamodel-Based Design Optimization}

Detailed simulation models of structures are often computationally expensive to evaluate. Metamodel-based design optimization (MBDO), in which metamodels are used for the evaluations, is an approach to significantly decrease the required computational effort. In fact, direct optimization where the detailed simulation models are used directly for the evaluations, require in many cases more computational resources than are available.

A metamodel is an approximation of a response from a detailed simulation model and is thus a kind of surrogate model that take little time to evaluate. Metamodels are developed based on a series of evaluations of the detailed models, either iteratively during the course of the optimization process, or before the solution of the optimization problem starts. Many different approaches exist to develop metamodels and they can either interpolate or approximate the dataset used to construct the model. Additionally, they can be simple and valid only over a small portion of the design space, or more complex and intended to capture the response over the complete design space. Interpolating metamodels might intuitively be more appealing for deterministic simulations. However, interpolating metamodels are not necessarily better than approximating ones at predicting the response between the simulated designs. Further, interpolating metamodels capture the numerical noise, while approximating ones can smooth it out.

Metamodel-based design optimization is mainly suitable for problems with a limited number of continuous or ordered discrete design variables, i.e. many structural size and shape optimization problems. However, the metamodels add an extra source of error to the optimization process and the success of MBDO is highly related to the accuracy of the metamodels, i.e. how well the metamodels can predict the responses.

Metamodel-based design optimization is covered in more detail in Chapter 3. 


\subsection{Optimization Algorithms}

The solution to the optimization problem in Equation (2.1) is usually found through an iterative search process and many different algorithms can be used depending on the characteristics of the problem.

Gradient-based optimization algorithms make use of information on the gradients of the objective and constraint functions to find the optimum solution. These algorithms are efficient, can solve problems with many design variables, and usually require little problem-specific parameter tuning. However, multiple searches from different starting designs are normally needed to find the global optimum. Additionally, these algorithms have difficulty solving discrete optimization problems and may be susceptible to numerical noise.

In many non-linear applications, as vehicle crashworthiness, gradients are difficult or impossible to evaluate in FE models. Stochastic optimization algorithms only use function values, not gradients, and can then be used instead. Additionally, these algorithms have a better chance than gradient-based ones to find the global or near global optimum, they are robust, and they are well suited for discrete optimization problems. However, stochastic optimization algorithms usually need problem-specific parameter tuning and they normally require more function evaluations than gradient-based algorithms. The large number of evaluations needed is not a concern in MBDO, since evaluations using metamodels take little time, and stochastic optimization algorithms are therefore often used for MBDO.

Many stochastic optimization algorithms are population-based, i.e. a set of design points search for the optimum solution. These algorithms are especially suitable for multiobjective optimization problems since a set of Pareto optimal points can be found in one single optimization run.

Stochastic optimization algorithms are typically inspired by some phenomenon from biology or physics. As an example, evolutionary algorithms including genetic algorithms (Holland 1992) and evolution strategies (Beyer and Schwefel 2002), try to mimic biological evolution and are inspired by Darwin's idea of survival of the fittest. Simulated annealing (SA), on the other hand, mimics the metallurgical annealing process and exploits the analogy with a metal that cools and freezes into a minimum energy crystalline structure (Kirkpatrick et al. 1983).

In SA, the objective function of the optimization problem is seen as the internal energy of the metal during annealing. The idea is to start at a high temperature, which is slowly reduced so that the system goes through different energy states in the search of the lowest state representing the global minimum of the optimization problem. As the temperature is decreased, new candidate solutions are iteratively selected and compared to the current solution. The candidate solutions can, at a certain probability, be accepted as the current 
solution even if they are worse than the previous one, i.e. have higher energy. A new worse solution is more likely to be chosen early in the search when the temperature is high and if the difference in energy is small. Simulated annealing hence goes from initially being similar to random search, with the aim of finding the region of the global optimum, to only accepting better solutions in the end, in order to locate the minimum more exactly.

When annealing metals, the initial temperature must not be too low and the cooling must be done sufficiently slowly to avoid that it gets stuck in a meta-stable non-crystalline state representing a local minimum of energy. The same principles apply to SA in the process of finding the solution to an optimization problem. The strength of the SA algorithm is its ability to deal with highly non-linear, chaotic, and noisy objective functions, as well as a large number of constraints. Different modifications to the original algorithm have been proposed to improve the speed of convergence. One of these is the very fast simulated reannealing algorithm, also known as adaptive simulated annealing (ASA) (Ingber 1996), which has been used in this thesis.

\subsection{Topology Optimization}

Topology optimization is used to identify the best possible material distribution within a certain design volume. Normally, the design volume is continuous and the optimal load paths within this volume to carry the applied loads are sought. However, topology optimization can also be used for discontinuous design domains, and hence for optimization of spot weld layouts.

One common topology optimization approach is to consider fictitious material densities of all the finite elements in the design volume as variables, i.e. the number of design variables is typically very large. A relationship between the fictitious material densities and the mechanical properties is defined and the optimal density distribution due to the applied loads is computed while fulfilling the constraints. Intermediate densities are normally penalized during the optimization process to obtain a clear result where elements are either empty or full of material. This method was introduced by Bendsøe (1989) and is usually called SIMP, solid isotropic material with penalization. The traditional approach to solve topology optimization problems involves gradients and can thus only be used for linear-static problems and not for general MDO. Topology optimization procedures for non-linear structures have been proposed, see e.g. Patel et al. (2009), but are not yet commonly used within industry. 
CHAPTER 2. STRUCTURAL OPTIMIZATION 


\section{Metamodel-Based Design Optimization}

A metamodel is a mathematical description created based on a dataset of input and the corresponding output from a detailed simulation model, i.e. a model of a model, see Figure 3.1. Once the model is developed, the approximate response at any sample location can be evaluated and used in metamodel-based design optimization (MBDO). A general form of a metamodel is

$$
y(\mathbf{x})=\hat{y}(\mathbf{x})+\varepsilon
$$

where $y(\mathbf{x})$ is the true response obtained from the detailed model, $\hat{y}(\mathbf{x})$ is the approximate response from the metamodel, and $\varepsilon$ represents the approximation error. Traditionally, metamodels are simple polynomials, but other metamodels that can capture complex responses increase in popularity. The process of where to place the fitting samples in the design space is often called design of experiments (DOE). The term can also refer to the resulting sampling plan (Queipo et al. 2005).

a)

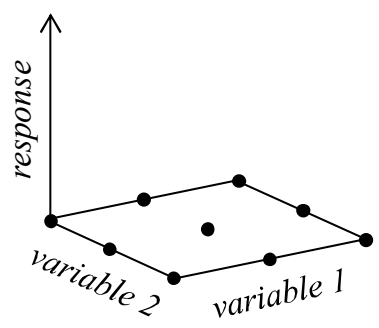

b)

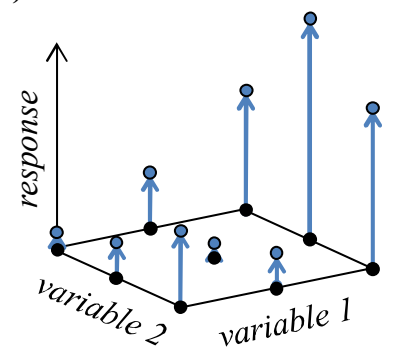

c)

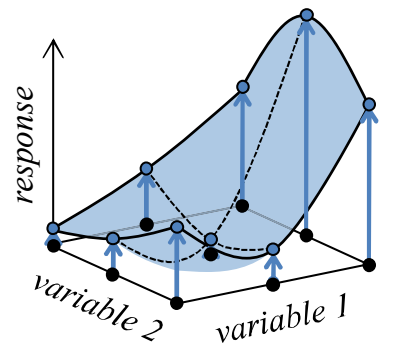

Figure 3.1 The concept of building a metamodel of a response depending on two design variables: a) design of experiments, b) function evaluations, and c) metamodel.

The number of simulations needed to build a metamodel depends largely on the number of design variables. Variable screening is therefore often used to identify the important variables of the problem in order to reduce its size and thereby decrease the required number of detailed simulations. Several screening methods exist, see e.g. Viana et al. (2010). One of the simplest techniques evaluates the effect of changing one variable at a time. It is 
an inexpensive approach, but it does not estimate the interaction effects between variables. Another simple and commonly used approach is based on analysis of variance (ANOVA) as described by Myers et al. (2008). In this method, a metamodel is fitted using regression analysis, for example a simple polynomial metamodel, and the coefficients for each term in the model are studied. An alternative is Sobol's global sensitivity analysis (GSA), which provides the total effect, i.e. main and interaction effects, of each variable (Sobol' 2001). This method can be used for arbitrary metamodels and includes the calculation of sensitivity indices, which can be used to rank the importance of the design variables.

There exist different MBDO strategies. One popular approach is the sequential response surface method (SRSM), in which simple polynomial metamodels, often linear ones, are used for the evaluations. The metamodels are built sequentially over a subregion of the design space, and the subregion is moved and reduced in size to close in on the optimum solution. Another approach is to build metamodels that should capture the response over the complete design space before the optimization starts. This approach requires flexible metamodels that can capture complex responses. Despite its simplicity, the SRSM can work remarkably well and outperform the second approach if the global metamodels have insufficient accuracy (Duddeck 2008). However, many iterations can be required to find the optimum solution for complex responses. The approach with global metamodels has the benefit of rendering a view of the complete design space, it is suitable for finding Pareto optimal solutions, and it is inexpensive to rerun optimizations, for example with changed constraint limits.

Many comparative studies have been made over the years to guide the selection of metamodel type, see e.g. Jin et al. (2001), Clarke et al. (2005), Kim et al. (2009), Li et al. (2010), and Backlund et al. (2012). Still, it is impossible to draw any decisive conclusions regarding the superiority of any type of metamodel. In contrast, there seem to be a consensus among scientists that a proper DOE for fitting global metamodels over a large design space with many variables should be space-filling. However, there exist many techniques to develop such DOEs. In this thesis, various space-filling DOEs are used in combination with different versions of neural network metamodels. These metamodel types are selected due to their flexibility and ability to capture complex responses.

\subsection{Design of Experiments}

Classical DOEs, as factorial designs, central composite designs, and $D$-optimal designs, focus on reducing the effect of noise in physical experiments. These designs tend to spread the samples around the border and only put a few samples in the interior of the design space. When the dataset is used to fit advanced metamodels, where interesting phenomena can be found anywhere in the design space, DOEs aiming to spread the samples over the complete design space is preferred. 


\subsubsection{Latin Hypercube Designs}

One popular space-filling design is the Latin hypercube design (LHD), which is a constrained random design initially proposed by McKay et al. (1979). To develop an LHD of $n$ samples for $k$ variables, the range of each variable is divided into $n$ non-overlapping intervals of equal probability. For each variable, one value from each interval is selected at random but with respect to the probability density in the interval. The $n$ values of each variable are then randomly combined with the values of the other variables so that $n k$ tuplets are formed, see Figure 3.2. A common variant is the median LHD, which has samples from the centre of the intervals.

A standard LHD might not have good space-filling properties, see Figure 3.3. To generate an improved space-filling design, the LHD can be taken as a starting design, which is then altered to optimize some criterion. One approach is to maximize the distances between samples. Such optimal LHDs are usually obtained from time-consuming optimization procedures. However, Viana et al. (2010) instead proposed to translate small building blocks, i.e. seed designs of one or more points, in the hyperspace to obtain an optimum or near optimum LHD without the use of formal optimization.

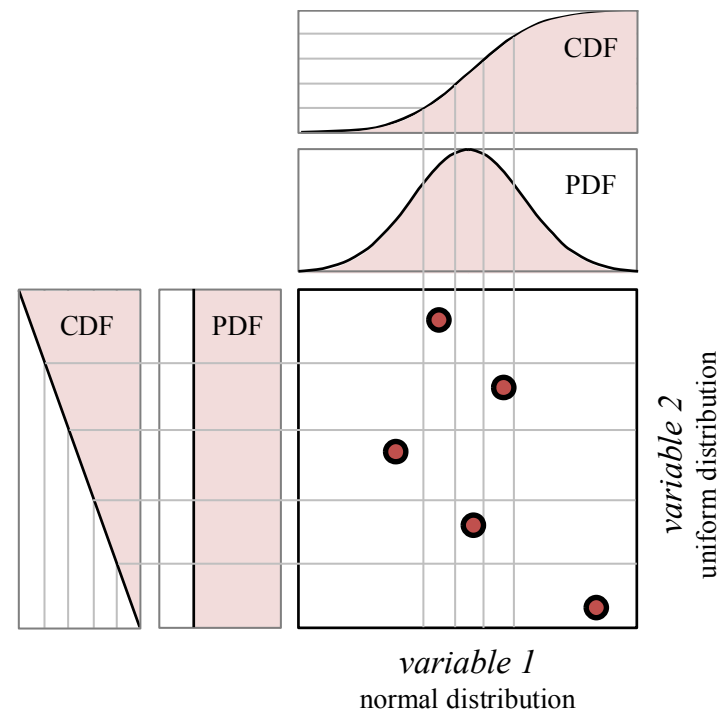

Figure 3.2 Latin hypercube design for two variables at five levels, one normally distributed variable and the other uniformly distributed.

\subsubsection{Distance-Based Designs}

Distance-based designs place samples based on a measure of distance. Maximizing the minimum distance between samples, results in a maximin design (Johnson et al. 1990). 
For small DOE sizes, this will generally result in samples lying on the exterior of the design space, and that the interior is filled as the number of samples becomes larger. Minimizing the maximum distance between samples, results in a minimax design. In this case, the samples will generally lie in the interior of the design space also for small DOEs.

A comparison between the two designs in two variables is provided in Figure 3.4. It can be seen that the maximin design fills the design space with spheres with maximum radius, i.e. no sample is too close to the other samples. The minimax design, instead, covers the design space with spheres with minimum radius, i.e. no sample is too far from the others.

The maximin and minimax designs have good space-filling properties. However, in contrast to LHDs, they can include samples with one or more identical variable values, i.e. poor projection properties, as seen when comparing Figure 3.3 and Figure 3.4.

a)

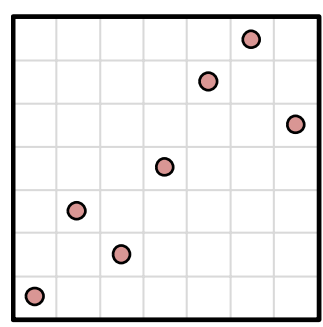

b)

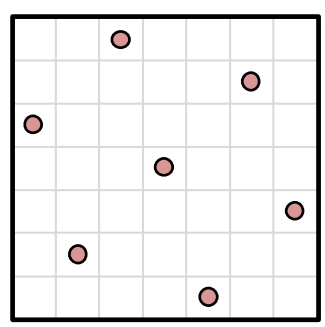

Figure 3.3 Two different median LHD with seven samples in two variables that have a) weak space-filling qualities, and b) better space-filling qualities.

a)

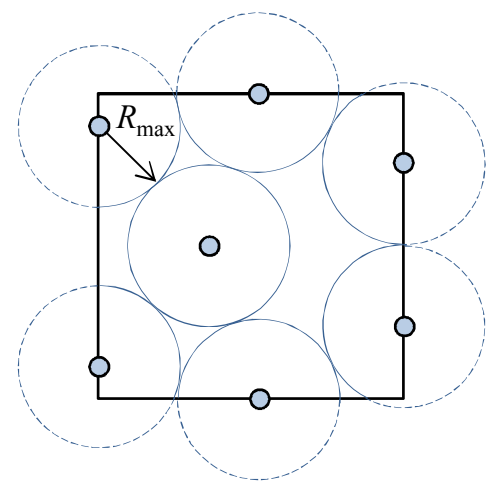

b)

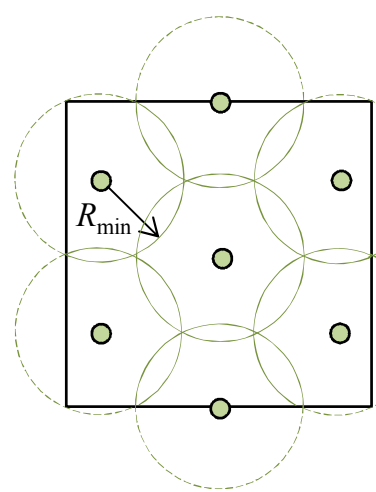

Figure 3.4 Comparison of maximin and minimax designs with seven samples in two variables: a) maximin, b) minimax. 


\subsubsection{Sequential Sampling}

To achieve sufficiently accurate metamodels without spending too much computational effort, the size of the DOE is often gradually increased through sequential sampling. When the metamodels are found to be satisfactory, they are used for optimization. In addition to the number of samples, also the distribution of them will influence the accuracy of the metamodels. If this is taken into account when deciding on new samples, sequential sampling can also lead to improved metamodels compared to when the same number of samples is selected at once.

A common sequential sampling technique is the maximin distance approach, where new samples are placed as far away from the existing samples as possible. This technique is exploring the design space and ensures that no important areas of the design space are overlooked. When the output from the simulations or information from previously fitted metamodels is used in the sequential sampling, the sampling is said to be adaptive and it exploits interesting areas of the design space. As an example, Kriging models provide an estimation of the prediction error at an unobserved point, and this information can be used to identify new samples, see e.g. Jin et al. (2002). For other models, where an estimate of the prediction error is not provided, cross validation can be used to obtain it.

An issue with adaptive sequential sampling techniques arises when several responses from one detailed simulation model are studied. Then, several metamodels are fitted to the same DOE, but ideally, different samples should probably be selected for the various responses.

\subsection{Metamodels}

Many techniques for developing advanced metamodels capable of capturing complex responses, i.e. suitable as global metamodels, have been proposed. Some of the more wellknown ones are Kriging (Sacks et al. 1989), radial basis functions (RBFs) (Hardy 1990), multivariate adaptive regression splines (MARS) (Friedman 1991), support vector regression (SVR) (Smola and Schölkopf 2004), and artificial neural networks (ANNs). It has been shown by Hornik et al. (1989) that certain ANNs can approximate any continuous function to any desired degree of accuracy. In theory, these metamodels thus have the flexibility to approximate very complex functions, as well as simple ones, which make them suitable for MDO. Two different versions of ANNs have been used in the present work and these are described in the following subsections.

\subsubsection{Feedforward Neural Networks}

Artificial neural networks are intended to respond to stimulus in a way similar to the biological nervous systems. They are composed of small computing elements, called neurons, which are assembled into an architecture that normally consist of an input layer, one or 
more hidden layers, and an output layer. In a general neuron, a biased and weighted sum of its input is transformed by a function to form its output, see Figure 3.5 This function is called activation or transfer function, and will vary depending on the type of ANN.

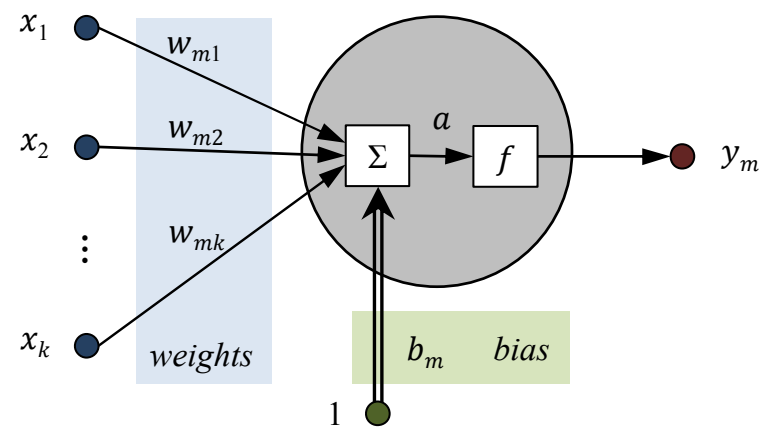

Figure 3.5 Illustration of neuron $m$ in a neural network.

One common neural network architecture is the feedforward neural network (FFNN), in which the information is only passed forward in the network, see Figure 3.6. The transfer function in the hidden layers of an FFNN is often a sigmoid function, i.e.

$$
f(a)=\frac{1}{1+e^{-a}}
$$

which is an S-shaped curve ranging from 0 to 1 . For neuron $m$, which has $k$ input,

$$
a=b_{m}+\sum_{i=1}^{k} w_{m i} x_{i}
$$

as described previously, where $b_{m}$ is the bias value and $w_{m i}$ the weight of input $x_{i}$. For the input and output layers, a linear transfer $f(a)=a$ is often used with bias added to the output layer, but not to the input layer. This means that a simple neural network with only one hidden layer of $M$ neurons can be of the form

$$
\hat{y}(\mathbf{x})=b+\sum_{m=1}^{M} w_{m} f(a)=b+\sum_{m=1}^{M} \frac{w_{m}}{1+e^{-\left(b_{m}+\sum_{i=1}^{k} w_{m i} x_{i}\right)}}
$$

where $b$ is the bias of the output neuron, $w_{m}$ is the weight for the connection between the $m^{\text {th }}$ hidden neuron and the output neuron, $b_{m}$ is the bias in the $m^{\text {th }}$ hidden neuron, and $w_{m i}$ is the weight for the connection between the $i^{\text {th }}$ input and the $m^{\text {th }}$ hidden neuron. For bounded output, a sigmoid transfer function is probably more suitable also for the output layer and Equation (3.4) needs to be modified accordingly. 


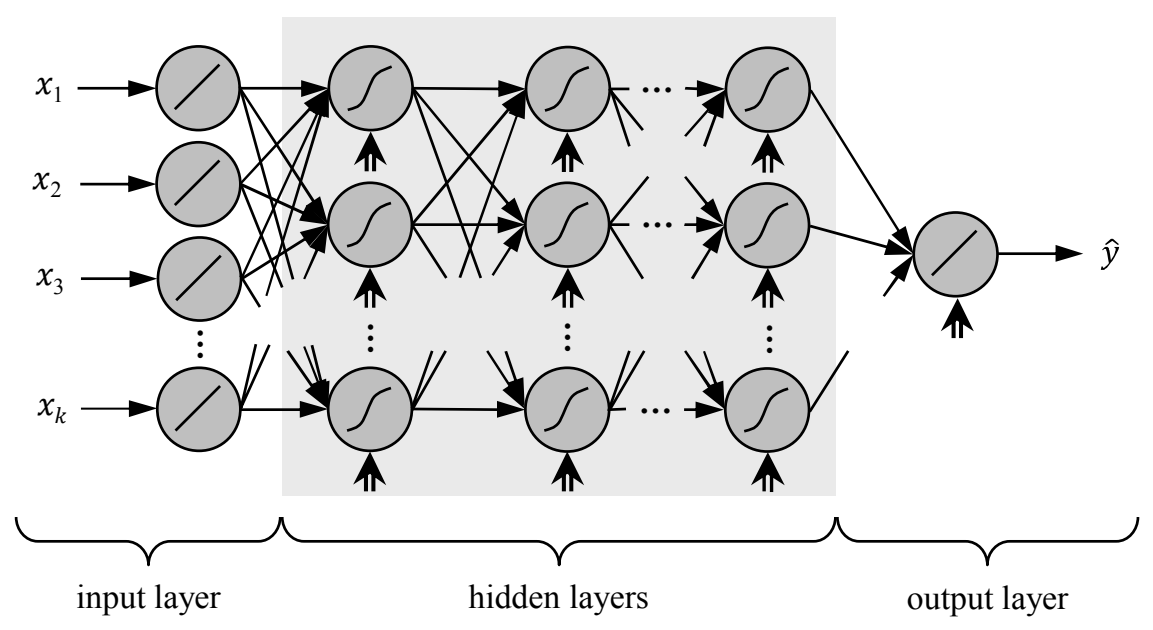

Figure 3.6 Illustration of a feedforward neural network architecture with multiple hidden layers.

There are two aspects in building an FFNN. The first one is to choose the architecture, i.e. the number of hidden layers and the number of neurons in them. The second one is to train the network to perform well with respect to the training set, i.e. determine the weights and biases. This is a non-linear optimization problem in which some error measure is minimized. The decision on the appropriate number of neurons in the hidden layers is not trivial. Generally, the correct number of neurons is determined experimentally, i.e. a number of candidate networks are constructed and the one judged to be the best is selected. Often, only one hidden layer is used.

If the number of hidden neurons is sufficiently large and the training optimization is run long enough, it is possible to drive the fitting error as close to zero as preferred. However, too small errors are not desirable since it can lead to overfitting and poor prediction capabilities of the metamodel. Given a fixed amount of training data, it is beneficial to reduce the number of weights and biases, as well as the size of them, in order to avoid overfitting. Regularization means that some constraints are applied to the construction of the ANN in order to reduce the prediction error in the final model. A fundamental problem is to balance between the goodness of fit and the choice of how strong the constraints forced on the model by regularization should be.

\subsubsection{Radial Basis Function Neural Networks}

Another common type of neural network, in addition to the FFNN, is the radial basis function neural network (RBFNN), which has transfer functions in the form of radial basis functions. An RBFNN has one single hidden layer built of non-linear radial units, each 
responding only to a local region of the design space. The input layer is linear and the output layer performs a biased weighted sum of the hidden layer units and creates an approximation over the entire design space. The RBFNN model is sometimes complemented with a linear part corresponding to additional direct connections from the input neurons to the output neuron.

Commonly used RBFs are Gaussian functions and Hardy's multiquadrics, i.e.

$$
f(a)=e^{-\left(\frac{a}{c}\right)^{2}}
$$

and

$$
f(a)=\sqrt{1+\left(\frac{a}{c}\right)^{2}}
$$

respectively, where $c$ is a shape parameter that controls the width of the RBF. The activation of the $m^{\text {th }} \mathrm{RBF}$, which has $k$ input, is determined by the Euclidean distance

$$
a=\sqrt{\sum_{i=1}^{k}\left(x_{i}-w_{m i}\right)^{2}}
$$

between the input $x_{i}$ and the RBF centres $w_{m i}$. This means that an RBFNN with $M$ hidden neurons, but without linear part, will be of similar form as Equation (3.4), but with a different transfer function.

The training of an RBFNN is generally done in two stages. First, the centres and widths of the radial units are set, and thereafter the output layer parameters are determined. Due to its form, RBFNNs can often be trained much faster than FFNNs. A classical approach is to set centres equal to all the fitting samples and use the same width parameter for all the RBFs. However, different choices might lead to improved models. To find a suitable number of RBFs and their parameters, it is possible to loop over several trial values and select the metamodel judged to be the best.

\subsection{Metamodel Validation}

Since a metamodel is an approximation of the true response, it is important to evaluate its accuracy, i.e. how well the metamodel represents the detailed simulation model. This can be done by studying residuals, i.e. the difference between the values from the detailed simulation model and the metamodel. If the residuals for the samples used for developing the metamodel are studied, a picture of the fitting error is obtained. For an interpolating 
metamodel, this error is zero. The fitting error is not a good measure of how well the metamodel will predict at unknown design samples. However, a view of the prediction error can be obtained if residuals for samples not included in the fitting set are studied. It is essential that this validation set is large enough and that it is spread over the design domain to provide a reliable picture of the accuracy. It is also important that the validation samples are not placed too close to the fitting samples, since that can lead to an overoptimistic evaluation of the metamodel accuracy (Iooss et al. 2010).

\subsubsection{Error Measures}

Several error measures can be evaluated based on the residuals, e.g. the maximum absolute error (MAE), the average absolute error (AAE), the mean absolute percentage error (MAPE), the mean squared error (MSE), and the root mean squared error (RMSE). These are defined as

$$
\begin{aligned}
& \text { MAE }=\max \left|y_{i}-\hat{y}_{i}\right|, \quad i=1, \ldots, n \\
& \mathrm{AAE}=\frac{\sum_{i=1}^{n}\left|y_{i}-\hat{y}_{i}\right|}{n} \\
& \mathrm{MAPE}=\frac{\sum_{i=1}^{n}\left|\frac{y_{i}-\hat{y}_{i}}{y_{i}}\right|}{n} \times 100 \% \\
& \mathrm{MSE}=\frac{\sum_{i=1}^{n}\left(y_{i}-\hat{y}_{i}\right)^{2}}{n} \\
& \mathrm{RMSE}=\sqrt{\frac{\sum_{i=1}^{n}\left(y_{i}-\hat{y}_{i}\right)^{2}}{n}}
\end{aligned}
$$

where $y_{i}$ and $\hat{y}_{i}$ are the true and the approximated responses, and $n$ is the number of samples. The AAE, MAPE, MSE and RMSE provide a measure of the overall accuracy, while the MAE is a measure of the local accuracy. RMSE is the most commonly used metric but it can be biased. If the dataset contains both high and low response values, it might be desirable to study MAPE instead, which is an error measure that is independent of the magnitude of the response.

Another common statistic is the coefficient of determination $R^{2}$, which is a measure between 0 and 1 that describes how well the metamodel captures the variability in the dataset. 


$$
R^{2}=1-\frac{\sum_{i=1}^{n}\left(y_{i}-\hat{y}_{i}\right)^{2}}{\sum_{i=1}^{n}\left(y_{i}-\bar{y}\right)^{2}}=\frac{\sum_{i=1}^{n}\left(\hat{y}_{i}-\bar{y}\right)^{2}}{\sum_{i=1}^{n}\left(y_{i}-\bar{y}\right)^{2}}
$$

where $n$ is the number of samples, $y_{i}$ and $\hat{y}_{i}$ are the true and approximated responses, and $\bar{y}$ represent the mean of the true responses. An $R^{2}$ value of 1 indicates a perfect fit.

\subsubsection{Cross Validation}

When it takes long time to evaluate the detailed simulation models and the available data is limited, it can be desirable to use the complete dataset for fitting the metamodels rather than potentially lower the accuracy by leaving out a part of the set for validation. In cross validation $(\mathrm{CV})$ the same dataset is used for fitting and validating the model. Different versions of $\mathrm{CV}$ exist, but the main idea is that metamodels are fitted repeatedly to the main portion of the dataset and the left out samples are used to calculate residuals. Small fitting sets, which are common in reality, are not suitable for CV (Stander et al. 2015). Data distribution could change considerably even when a small portion of the dataset is removed and used as a validation set. Additionally, the process of developing several metamodels can be computationally expensive. Although, for the special case when only one sample is left out at a time, called leave-one-out $\mathrm{CV}$, an estimation of the prediction error can be inexpensively computed for some metamodels, e.g. polynomial (Myers et al. 2008), Kriging (Martin and Simpson 2004), and RBF models (Goel and Stander 2009).

\subsubsection{Generalized Cross Validation}

Overfitting generally occurs when a model is excessively complex, i.e. when it has too many parameters relative to the number of samples in the fitting set. Measures of goodness of fit have therefore been developed that take both the residual error and the model complexity into account. One of these methods is generalized cross validation (GCV). Metamodels of different complexity are then fitted to the same dataset and the model with the lowest GCV-error is chosen as the one with the appropriate complexity (Stander et al. 2015). For a metamodel with a mean squared fitting error MSE, the corresponding GCVerror is defined as

$$
\mathrm{MSE}_{\mathrm{GCV}}=\frac{\mathrm{MSE}}{\left(1-\frac{v}{n}\right)^{2}}
$$

where $n$ is the number of fitting samples, which should be large, and $v$ is the number of effective model parameters. For linear or unbiased models without regularization, $v$ is the number of model parameters. Otherwise, for example for neural network models, $v$ should be the number of effective model parameters, which can be estimated in different ways. 


\section{Multidisciplinary Design Optimization}

Multidisciplinary design optimization (MDO) is used to optimize a design taking several disciplines into account simultaneously. All evaluations required for the different disciplinary load cases are performed by subspace analyzers. Some of the variables in an MDO problem are related to several subspaces, while others are unique to one specific subspace. These variables are called shared and local variables, respectively. In the general case, the subspaces are coupled, i.e. output from one subspace is needed as input to other subspaces. The couplings between subspaces are handled by coupling variables and an iterative approach is needed to find a consistent solution, i.e. a solution in balance. If the objective function $f$ is assumed to be computed from a combination of data $f_{j}$ from $n$ different subspaces, and the constraints $\mathbf{g}$ are assumed to be separable, i.e. belong only to one subspace, the resulting MDO problem can be formulated as

$$
\begin{array}{cl}
\min _{\mathbf{x}} & f\left(f_{1}\left(\mathbf{x}_{l 1}, \mathbf{x}_{s 1}, \mathbf{y}_{1 *}\right), f_{2}\left(\mathbf{x}_{l 2}, \mathbf{x}_{s 2}, \mathbf{y}_{2 *}\right), \ldots, f_{n}\left(\mathbf{x}_{l n}, \mathbf{x}_{s n}, \mathbf{y}_{n *}\right)\right) \\
\text { s.t. } & \mathbf{g}_{j}\left(\mathbf{x}_{l j}, \mathbf{x}_{s j}, \mathbf{y}_{j *}\right) \leq \mathbf{0}, \quad j=1,2, \ldots, n
\end{array}
$$

where $\mathbf{x}_{l j}$ and $\mathbf{x}_{s j}$ are the local and shared variables for subspace $j$, and $\mathbf{y}_{j^{*}}$ are the coupling variables input to subspace $j$.

Different approaches can be used to solve Problem (4.1). Methods suitable for large-scale problems aim to let the groups involved work concurrently and autonomously. To allow groups to work concurrently increases efficiency, as human and computational resources are used in parallel. Groups that work autonomously are not required to constantly share information with other groups. They can also govern the choice of methods and tools, and use their expertise to take part in design decisions. Multidisciplinary design optimization methods are either single-level or multi-level. The single-level methods have a central optimizer making all design decisions, while multi-level methods have a distributed optimization process. Hence, they are sometimes referred to as monolithic and distributed methods, respectively. Most multi-level methods were developed when MDO was applied to large problems in the aerospace industry involving several groups within a company. The intention was to distribute the work over many people and computers to compress calendar time for problems with coupled disciplines (Kodiyalam and SobieszczanskiSobieski 2001). However, multi-level methods complicate the solution process and to justify their use, the benefits must be greater than the cost. 
The disciplines in aerospace MDO problems are generally linked by both shared and coupling variables. As an example, due to the slender shapes of aeroplane wings, structural deformations are induced by aerodynamic forces. These deformations in turn affect the aerodynamics of the structure and hence the aerodynamic forces. The structural and aerodynamic disciplines are thus coupled. Subspaces in MDO studies of automotive structures are usually linked by shared variables, but there are less common coupling variables that must be considered. Therefore, the formulations for different MDO methods will henceforth be given without coupling variables.

\subsection{Single-Level Methods}

Single-level optimization methods solve MDO problems as a single optimization problem. The most common and basic single-level approach is the multidisciplinary feasible (MDF) formulation (Cramer et al. 1994). In this formulation, an optimizer requests the values of the objective and constraint functions for different sets of design variables from a system analyzer, which enforces multidisciplinary consistency from the subspace analyzers. The individual discipline feasible (IDF) formulation is an alternative approach proposed by Cramer et al. (1994), where multidisciplinary consistency is only enforced at optimum. Here, the subspace analyzers are decoupled as the optimizer sends the design variables and estimations of the coupling variables directly to the subspace analyzers. An additional constraint is introduced for each coupling variable to drive the solution towards multidisciplinary consistency at optimum. For MDO problems that lack coupling variables, the two formulations coincide, see Figure 4.1.

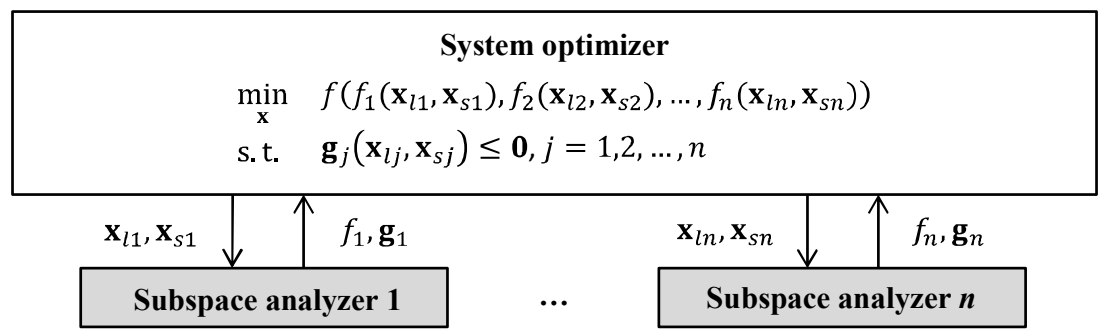

Figure 4.1 Single-level MDO formulation for a problem without coupling variables.

\subsection{Multi-Level Methods}

Many multi-level methods that decompose the original problem in various ways have been proposed, see e.g. Martins and Lambe (2013). One of the oldest methods is concurrent subspace optimization (CSSO) (Sobieszczanski-Sobieski 1988), which iteratively distri- 
bute each shared variable to the subspace it affects the most before all subspaces are optimized. Another well-known method is bilevel integrated system synthesis (BLISS) (Sobieszczanski-Sobieski et al. 1998), which iteratively optimize all subspaces with respect to the local variables before a system optimizer finds the best design with respect to the shared variables. However, it has been reported that both methods are ineffective and variants of the original formulations have been proposed (Martins and Lambe 2013).

An early description of collaborative optimization (CO) was published by Kroo et al. (1994), and it was further refined by Braun (1996). The method has been tested on different MDO problems, mostly in the design of aerospace systems, but also for weight reduction of a car (Xue et al. 2016). In CO, the system optimizer handles target values of the variables. The subspaces are given local copies of these variables, which they can change during the optimization process. The local copies converge towards the target values at optimum, i.e. a consistent design is obtained. The system optimizer minimizes the global objective function subject to constraints that ensure a consistent design. The subspace optimizers minimize the deviation from consistency subject to local constraints. A schematic picture of the $\mathrm{CO}$ formulation without coupling variables is given in Figure 4.2. Target values of the local variables are only set if they directly influence the global objective, otherwise the formulation can be simplified as shown in Paper I.

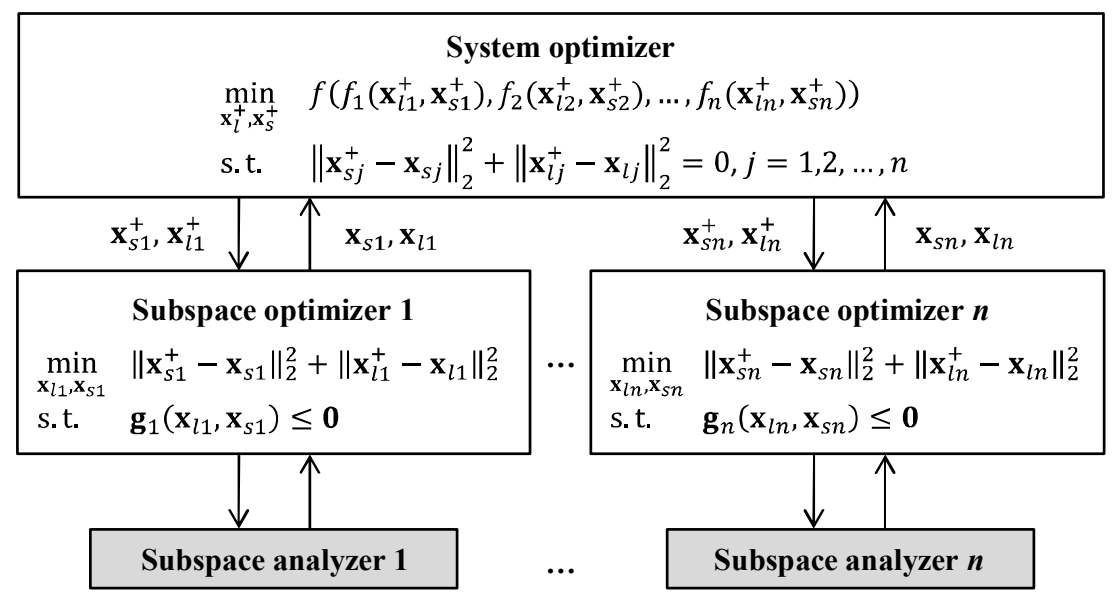

Figure 4.2 $\mathrm{CO}$ formulation for a problem without coupling variables, where the superscript + denotes target values.

There are a number of numerical problems associated with $\mathrm{CO}$ when used in combination with gradient-based algorithms. These often result in slow or no convergence. Many different modifications have therefore been proposed, including employing gradient-free optimization algorithms in combination with metamodels. Another solution is enhanced 
collaborative optimization (ECO) (Roth 2008), in which the roles of the system and the subspace optimizers are reversed, i.e. the system optimizer minimizes system inconsistency, while the subspaces minimize the system objective. The subspaces are subject to local constraints as well as to linearized versions of the constraints in the other subspaces. The inclusion of the latter constraints provides a direct understanding of the preferences of the other subspaces, but the complexity of ECO is a major drawback.

Analytical target cascading (ATC) was developed for automotive applications (Kim 2001, Kim et al. 2003, Michalek and Papalambros 2005). It was originally intended as a product development tool for propagating targets, i.e. convert targets on the overall system to targets on subsystems. However, it can also be used for optimization if the targets are unattainable. The method has mainly been applied to automotive design problems, see e.g. Kim et al. (2002), Han and Papalambros (2010), and Kang et al. (2014). Different ATC formulations exist, but the main idea is that the objective function on all levels consists of two terms, one that is part of the global objective and one that ensures consistency. In each of these problems, there are local variables and local constraints. The shared and coupling variables are handled in a fashion similar to $\mathrm{CO}$, i.e. an upper level optimizer has target values and a lower level optimizer has local copies of these variables. A schematic picture of a two-level ATC version without coupling variables adapted from Tosserams et al (2006) is given in Figure 4.3. The system optimizer can also be only coordinating, i.e. there is no system analyzer and $f_{0}$ and $\mathbf{g}_{0}$ vanish.

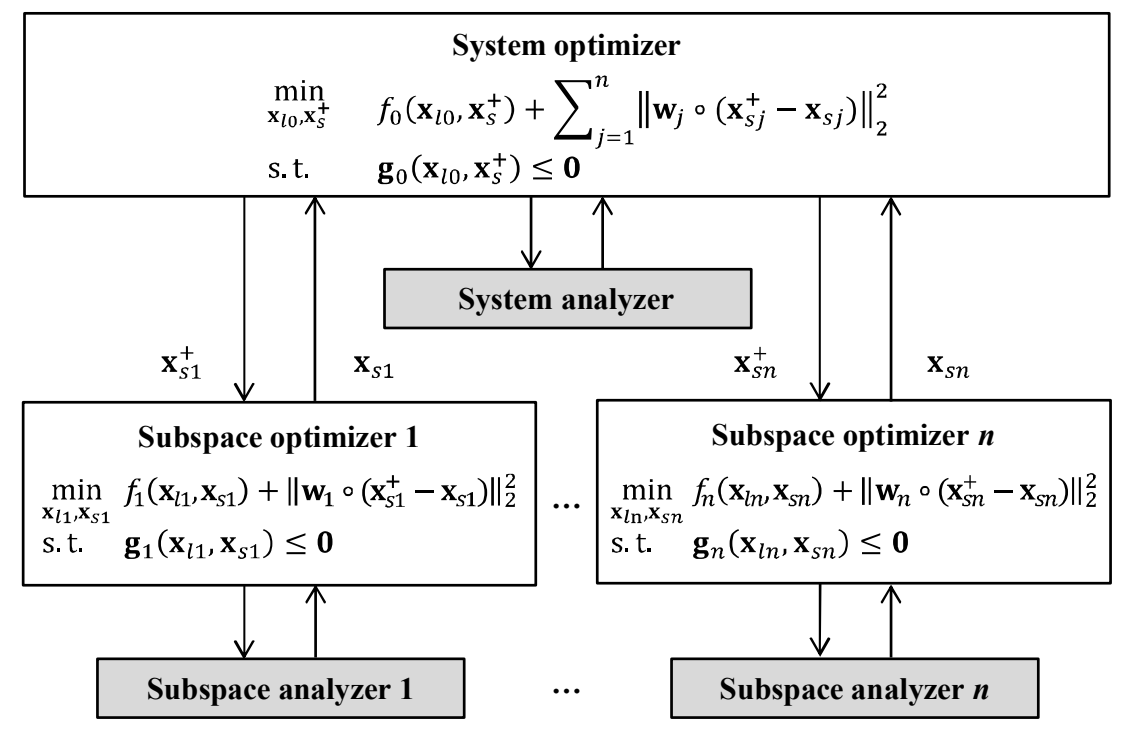

Figure 4.3 ATC formulation in two levels for a problem without coupling variables, where the superscript + denotes target values, $\mathbf{w}_{j}$ for $j=1, \ldots, n$ are penalty weights, and the symbol $\circ$ denotes a term-by-term multiplication of vectors. 


\subsection{An MDO Process for Automotive Structures}

Vehicle development has gone from designing and testing a series of prototypes to a process where most aspects of the development are performed with help of computers and physical testing is kept to a minimum. Models of the vehicle and its components are created using computer aided design $(\mathrm{CAD})$ and the performance of them is tested through computer aided engineering (CAE). Hence, the development process has changed from being hardware-based to being CAE-based. During today's virtual development, the designs are continuously developed by different groups in parallel, see Figure 4.4. At certain times, the CAD models are synchronized and simulation models are built. Virtual assessments of the vehicle can then be made based on simulation results and other available information. Afterwards, the development continues and discovered issues are resolved jointly by the designers and the simulation experts.

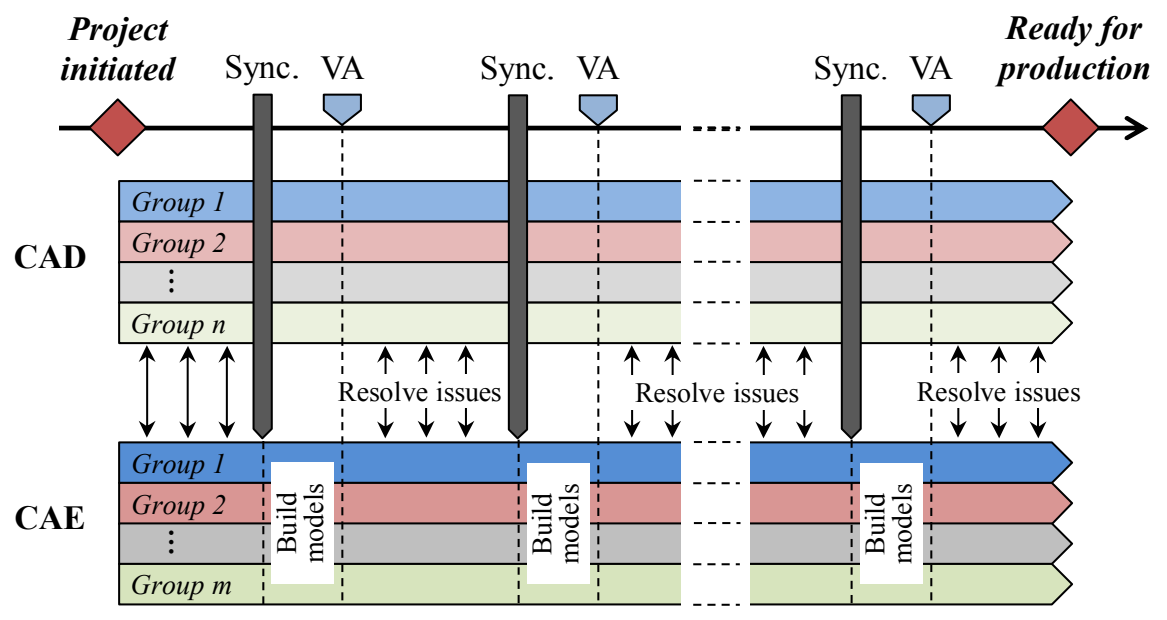

Figure 4.4 Schematic illustration of virtual development from Paper II, where VA indicates the virtual assessments.

To be able to implement MDO into an automotive product development process and use it for large-scale applications, the groups involved need to work concurrently and autonomously. This need is the motivation for the development of multi-level MDO methods. However, most multi-level methods were initially developed for direct optimization of coupled disciplines, and the gain in concurrency and autonomy is obtained at the cost of added complexity. The methods can be complicated both to implement and use. A multilevel MDO problem can also become less transparent than the corresponding single-level problem. One example is when the subspace objective functions do not reflect the objective of the overall problem, which makes it difficult for the individual groups to 
grasp the global goal of the optimization process. Some of the methods are also associated with numerical problems when used in combination with gradient-based optimization algorithms. Furthermore, multi-level MDO methods generally require more computational resources than single-level methods (Martins and Lambe 2013) and have turned out to be inefficient for loosely coupled automotive structural problems (Xue 2016).

During optimization of automotive structures, it is often necessary to use metamodels to reduce the required computational effort. It is possible to use metamodels in both singlelevel and multi-level MDO methods, but the effects are more interesting and important in combination with single-level methods. The effect of increased computational efficiency is obtained regardless of solution method. However, the drawback of limited autonomy and concurrency associated with single-level methods can be relieved by the introduction of metamodels. In MBDO, the main computational effort is spent when the metamodels are built. The groups involved can then work concurrently and autonomously using their preferred methods and tools. The issue of not participating in design decisions when using single-level methods can also partly be compensated for by involving the different groups in the setup of the optimization problem and in the assessment of the results. However, since the optimization is done on a system level, the individual groups cannot govern the choice of optimization methods and tools. In addition, groups that have inexpensive simulations either have to create metamodels and introduce an unnecessary source of error, or let the central optimizer call their analyzers directly and give up more of their autonomy. Despite these drawbacks, a single-level method in combination with the use of global metamodels is often the most convenient way to solve MDO problems for automotive structural applications that involve computationally expensive simulations. The complexity of the multi-level methods and lack of readily available software make them a less attractive alternative.

Consequently, it can be concluded that a suitable approach to implement large-scale MDO of automotive structures within current product development would be to perform MBDO with global metamodels. Actually, this is a method that can be used for both single- and multidisciplinary design optimization, and it can include robustness considerations if desired. The steps of the process are depicted in Figure 4.5, and described in more detail in Paper II. This process fits into the organization of automotive companies since existing groups of disciplinary experts can work concurrently and autonomously on the most timeconsuming steps of the process, i.e. step 2, 3, 4, and 6. An optimization expert should be responsible for the optimization, while the setup of the problem and the assessment of the results should be joint efforts. The described process fits a CAE-based development process since validated simulation models of the same status are available for all load cases at the virtual assessments. Hence, a suitable time to perform MDO studies will be shortly after these virtual assessments, in parallel with the regular development and problem solving. The outcome from the MDO studies can then be implemented together with all other design changes into the $\mathrm{CAD}$ models before the next synchronization. 


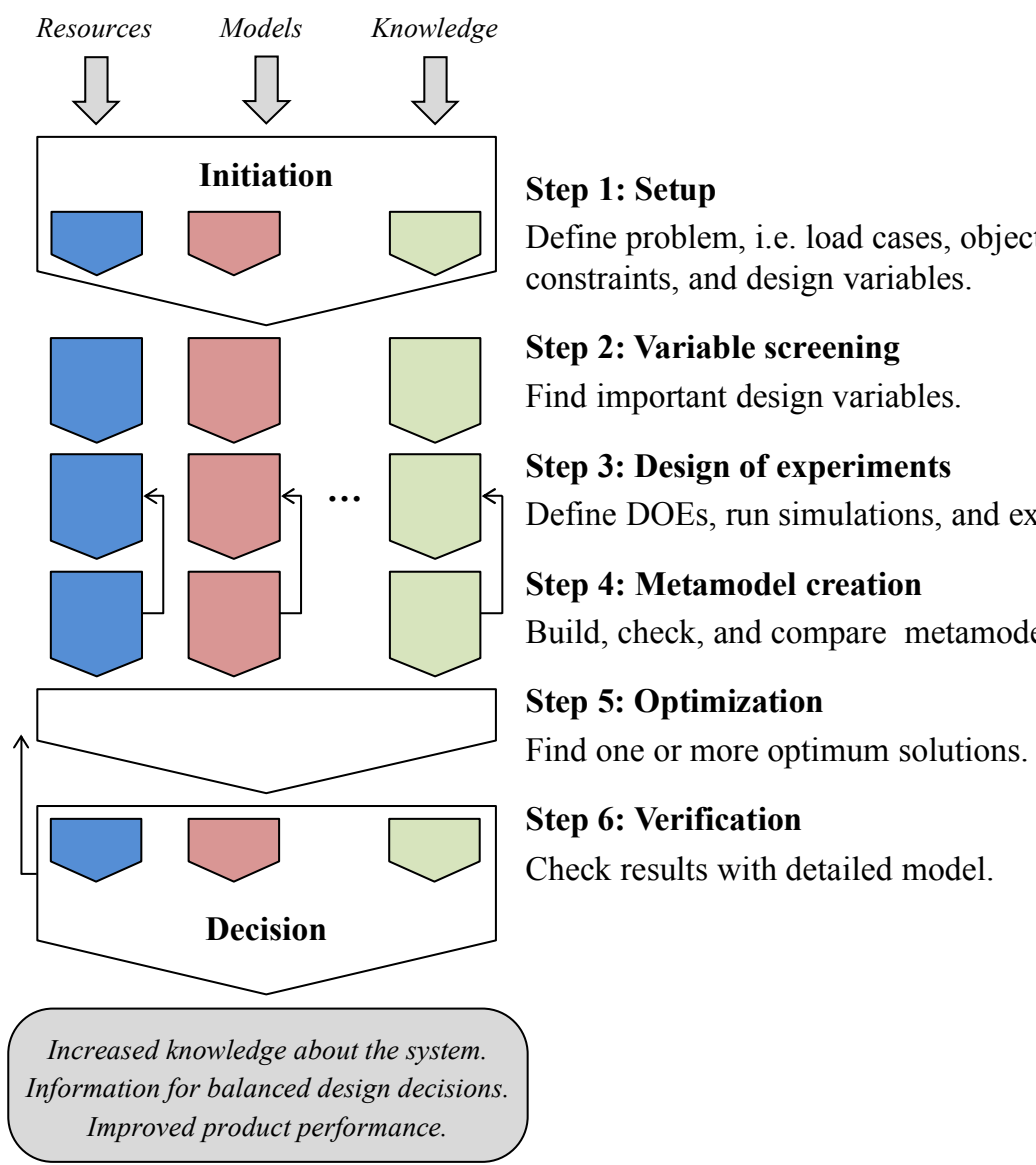

Figure 4.5 Process for MDO in which different disciplinary experts are responsible for the tasks indicated by colour and an optimization expert is responsible for the other tasks. 
CHAPTER 4. MULTIDISCIPLINARY DESIGN OPTIMIZATION

28 


\section{Support Vector Machines}

Classification is the problem of identifying to which category a new sample belongs, based on a training dataset of samples whose category membership is known. Support vector machines (SVMs) (Cortes and Vapnik 1995, Gunn 1998) are a class of machine learning techniques that can be used for classification or regression. The regression version is often called support vector regression (SVR) and can be used to develop metamodels. Many different classification techniques exist, but SVMs are used in this thesis due to their flexibility to define non-linear decision boundaries that optimally separate two classes of samples. Additionally, the boundaries constructed using SVMs can represent several disjoint regions in the design space.

\subsection{SVM Decision Boundary}

In the simplest case, an SVM separates the $n$ training samples $\mathbf{x}_{i}$ into two classes labelled $y_{i}= \pm 1$ using a hyperplane, or in the case of only two variables, a straight line. Note that $y_{i}$ in SVM classification terminology is the class label and not the response value of $\mathbf{x}_{i}$. The basic idea of the SVM algorithm is to maximize the distance between two parallel hyperplanes that pass through at least one training sample of each class, called support vectors. The optimum decision boundary $s(\mathbf{x})$ is half way between these parallel hyperplanes,

$$
s(\mathbf{x})=\mathbf{w} \cdot \mathbf{x}+b=0
$$

where $\mathbf{w}$ is the vector of hyperplane coefficients, $\mathbf{x}$ is a point in the design space, and $b$ is the bias.

The perpendicular distance between the hyperplanes is called the margin and is equal to $2 /\|\mathbf{w}\|$. The optimization problem to find the SVM decision boundary can be written as

$$
\begin{array}{ll}
\min _{\mathbf{w}, b} & \frac{1}{2}\|\mathbf{w}\|^{2} \\
\text { s.t. } & y_{i}\left(\mathbf{w} \cdot \mathbf{x}_{i}+b\right) \geq 1, \quad i=1, \ldots, n
\end{array}
$$

where the constraint defines that none of the $n$ training samples are allowed within the margin, see Figure 5.1. 


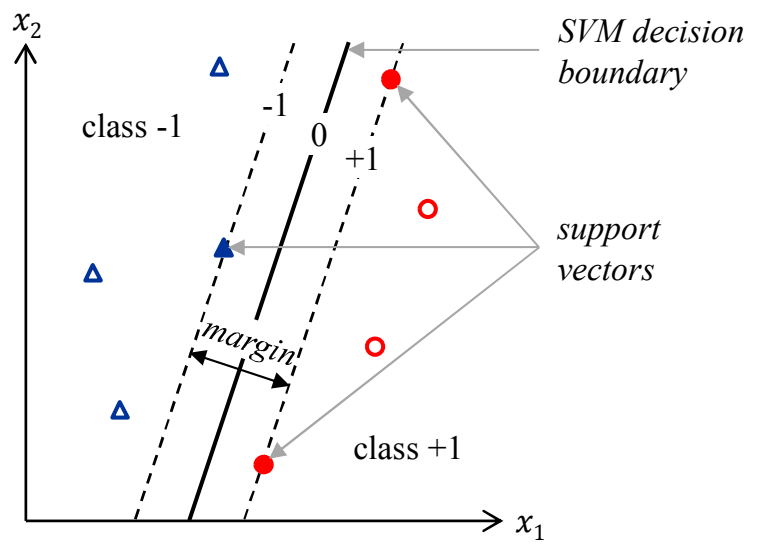

Figure 5.1 Linear SVM decision boundary.

Problem (5.2) is not always feasible and a larger margin might be obtained if some samples are allowed to be misclassified. Therefore, the soft-margin SVM, as suggested by Cortes and Vapnik (1995), relaxes the constraint through the introduction of slack variables $\xi_{i}$ and modifies the objective function to the following optimization problem:

$$
\begin{array}{ll}
\min _{\mathbf{w}, b, \xi} & \frac{1}{2}\|\mathbf{w}\|^{2}+C \sum_{i=1}^{n} \xi_{i} \\
\text { s.t. } & y_{i}\left(\mathbf{w} \cdot \mathbf{x}_{i}+b\right) \geq 1-\xi_{i} \\
& \xi_{i} \geq 0, \quad i=1, \ldots, n
\end{array}
$$

The penalty coefficient $C>0$ is referred to as the misclassification cost and defines the relative importance of maximizing the margin and minimizing the amount of slack.

Problem (5.3) is traditionally solved in its dual form and $C$ then becomes the upper bound for the Lagrange multipliers $\alpha_{i}$, see Gunn (1998).

$$
\begin{array}{ll}
\min _{\boldsymbol{\alpha}} & \frac{1}{2} \sum_{i=1}^{n} \sum_{j=1}^{n} \alpha_{i} \alpha_{j} y_{i} y_{j}\left(\mathbf{x}_{i} \cdot \mathbf{x}_{j}\right)-\sum_{k=1}^{n} \alpha_{k} \\
\text { s.t. } & \sum_{i=1}^{n} y_{i} \alpha_{i}=0 \\
& 0 \leq \alpha_{i} \leq C, \quad i=1, \ldots, n
\end{array}
$$

In the general case, the training samples need not be linearly separable in the input space, but can be so in a higher dimensional space, known as the feature space, see Figure 5.2. Since the mapping between the spaces will only appear within an inner product in 
Problem (5.4), the resulting function can be replaced by a kernel function $K$ (Boser et al. 1992). This modification, known as the kernel trick, allows for a non-linear decision boundary in the input space and results in the following optimization problem:

$$
\begin{array}{ll}
\min _{\boldsymbol{\alpha}} & \frac{1}{2} \sum_{i=1}^{n} \sum_{j=1}^{n} \alpha_{i} \alpha_{j} y_{i} y_{j} K\left(\mathbf{x}_{i}, \mathbf{x}_{j}\right)-\sum_{k=1}^{n} \alpha_{k} \\
\text { s.t. } & \sum^{n} y_{i} \alpha_{i}=0 \\
& 0 \leq \alpha_{i} \leq C, \quad i=1, \ldots, n
\end{array}
$$

Solving Problem (5.5) determines the Lagrange multipliers $\alpha_{i}$ and gives the SVM decision boundary as

$$
s(\mathbf{x})=\sum_{i=1}^{n_{s v}} \alpha_{i} y_{i} K\left(\mathbf{x}_{i}, \mathbf{x}\right)+b=0
$$

where $n_{s v}$ is the number of support vectors and

$$
b=-\frac{1}{2} \sum_{i=1}^{n_{s v}} \alpha_{i} y_{i}\left[K\left(\mathbf{x}_{i}, \mathbf{x}^{+}\right)+K\left(\mathbf{x}_{i}, \mathbf{x}^{-}\right)\right]
$$

The bias is here computed using two support vectors, $\mathbf{x}^{+}$and $\mathbf{x}^{-}$, which is any sample from each of the two classes. The class of a sample is given by the sign of $s(\mathbf{x})$.
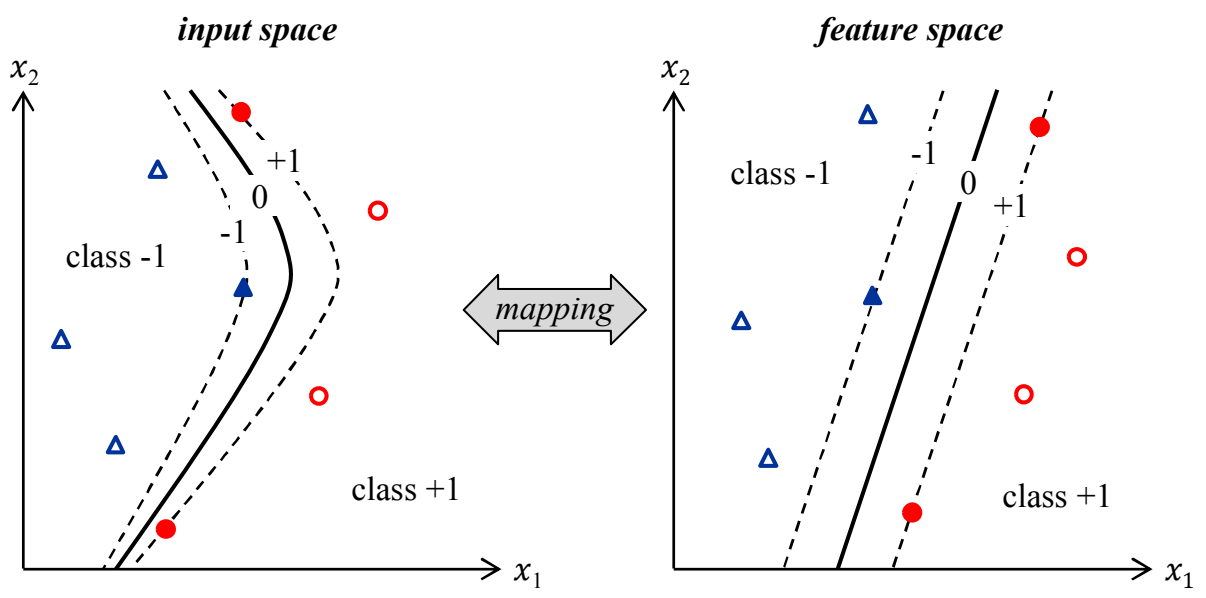

Figure 5.2 Mapping between input space and feature space. 
Different kernel functions can be used (Gunn 1998) and popular choices include polynomials and radial basis functions (RBFs). The Gaussian RBF kernel is used in this thesis due to its ability to form different decision boundaries and it is defined as

$$
K\left(\mathbf{x}_{i}, \mathbf{x}\right)=e^{-\frac{\left\|\mathbf{x}_{i}-\mathbf{x}\right\|^{2}}{2 \sigma^{2}}}
$$

where $\mathbf{x}_{i}$ is a support vector and $\sigma$ is the width parameter of the Gaussian kernel, which influences the shape of $s(\mathbf{x})$. A small value of $\sigma$ results in an irregular decision boundary with large curvature locally, while a large value results in a smoother surface.

It is important to select appropriate values for the parameters used in the SVM, for example reasonable values for $C$ and $\sigma$ (Ben-Hur 2010). This is normally done by testing different values and selecting the combination that performs best.

\subsection{SVMs to Improve Metamodel Accuracy}

Responses from impact simulations are often discontinuous due to bifurcations caused by instabilities or near contact situations. To develop accurate metamodels for such responses is a challenge, which to a large extent has hindered the execution of efficient large-scale optimization studies including crash load cases. However, as shown in Paper IV and V, the accuracy can be improved if the border between regions with different types of deformation behaviour is identified through an SVM, and this information is used when developing the metamodels.

One alternative is to split the design space and fit separate metamodels for the different regions. Another option is to add estimated guiding samples along the border to help a single metamodel better capture the discontinuity. The first approach results in a discontinuous metamodel and is only suitable for responses with a step-like behaviour. The second approach is more general and will usually improve the accuracy for discontinuous responses without decreasing it for smooth ones. Both approaches should preferably be combined with an SVM-based sequential sampling technique. Samples are then added on the SVM decision boundary to locate it more correctly (Basudhar 2011). Additionally, samples should be added elsewhere to improve the metamodel accuracy for the different types of deformation behaviour. All samples can be placed as far from the existing samples as possible. This type of combined exploring and exploiting sequential sampling technique can be beneficial also for more traditional metamodelling approaches. Compared to purely space-filling DOEs, where samples are evenly distributed in the design space, it results in a DOE with more samples in areas assumed to have large differences in response values between nearby samples.

The position of the estimated guiding samples can be defined in various ways. A general method that works for responses that are functions of many variables is to locate a pre- 
defined number of samples, for example three times the number of variables, on the decision boundary as far from the existing samples as possible, i.e. in the same way as the exploiting samples are selected in the SVM-based sequential sampling.

If the different types of deformation behaviour result in different levels of responses, i.e. the responses are separable, the class labels for the SVM training can be assigned based on a response limit value. This value can be selected in several ways as shown in Figure 5.3. If the responses are non-separable, the classification of the training set must be done differently, for example by visual inspection of deformation plots. For the case with separable responses, it is natural to assign the same response value to the estimated guiding samples as was used for defining classes. For the case with non-separable responses, the response value for the estimated guiding samples can be chosen based on identifying the largest response variation occurring in the training set, see Figure 5.4.

a)

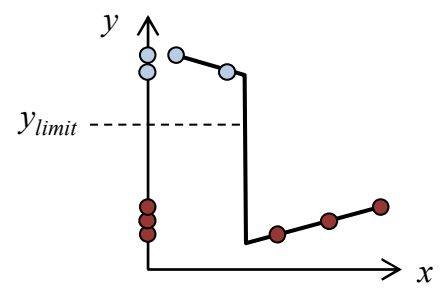

c)

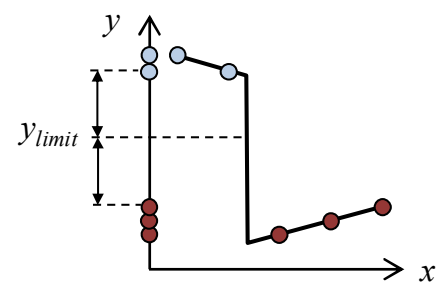

b)

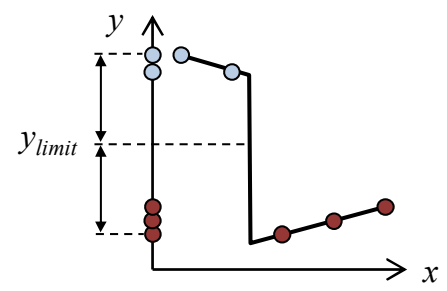

d)

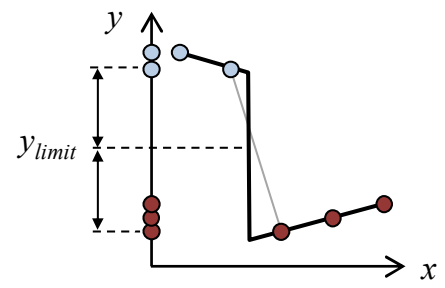

Figure 5.3 Illustration of different ways to define response limit values for a onedimensional case with separable responses: a) fixed value, b) response range, c) largest step, and d) steepest slope. 


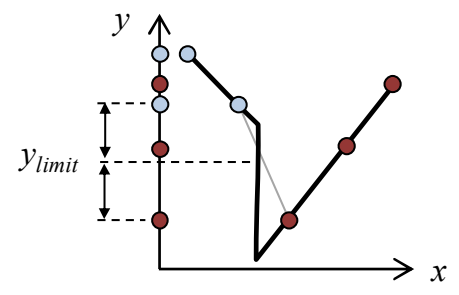

Figure 5.4 Illustration of a way to define the response value for the estimated guiding samples for a one-dimensional case with non-separable responses. 


\section{Summary of Appended Papers}

\section{6}

\section{Paper I}

\section{Multidisciplinary design optimisation methods for automotive structures}

Large-scale MDO problems typically involve several groups within a company who must work concurrently and autonomously to make the solution process efficient. In this paper, different MDO methods are assessed in relation to the characteristics of automotive structural applications. Both single-level and multi-level optimization formulations are considered. Multi-level optimization methods distribute the design process but are complex. When optimizing automotive structures, metamodels are often required to relieve the computational burden of detailed simulation models. The metamodels can be created by individual groups prior to the solution of the optimization problem, and therefore offer a way to distribute work. Consequently, it is concluded that a single-level method in combination with metamodels is the most straightforward way of implementing MDO into the development of automotive structures.

\section{Paper II}

\section{A metamodel-based multidisciplinary design optimization process for automotive structures}

Based on the findings from Paper I, a metamodel-based single-level MDO process intended for large-scale structural optimization with computationally expensive simulation models is presented and its implementation is briefly discussed. The process is flexible and it can easily fit into existing organizations and product development processes within the automotive industry due to the utilization of global metamodels. The process is demonstrated in an MDO example involving four load cases with the aim of minimizing mass by identifying the optimal thicknesses of 25 design variables. The process is proven to be efficient for the discussed example and it is concluded that improved designs can be obtained also for more complex industrial cases with comparable characteristics. 


\section{Paper III}

\section{Spot weld reduction methods for automotive structures}

Spot welds are regularly used to join steel sheets in automotive structures. Another interesting MDO task than the thickness optimization study successfully solved in Paper II is to find the appropriate number of spot welds for a structure. This paper presents three different methods of reducing the number of spot welds in automotive structures: rankingbased selection, topology optimization, and size optimization of a parameterized model. The third method can be performed according to the process presented in Paper II. The methods are compared in a simple example and it is found that the latter two methods have the best potential to reduce the number of spot welds. Topology optimization requires less preparation and computational effort compared to size optimization of a parameterized model. However, the method is primarily suitable for studies where load cases involving linear systems are judged to be most important. Otherwise, size optimization of a parameterized model is probably a better choice. The topology optimization approach is successfully demonstrated in a full-scale industrial application example and confirms that the method is useful within contemporary product development.

\section{Paper IV}

\section{Improving metamodel accuracy for structural responses including discontinuities}

The success of the MDO process presented in Paper II depends on the accuracy of the metamodels. Unfortunately, metamodels often fail to capture complex responses including discontinuities that are common in impact situations. The aim in this paper is to improve feedforward neural network metamodel accuracy for this type of responses without lowering it when representing smooth ones. The idea is to discriminate between different types of deformation behaviour in the design space using the decision boundary from a support vector machine. Three different approaches are tested; splitting the design space and fitting separate metamodels for the different regions, adding estimated guiding samples to the fitting set along the boundary before fitting a global metamodel, and using a special sequential sampling method. It is concluded that adding estimated guiding samples to the metamodel fitting set, combined with sequential sampling to locate the decision boundary, best meets the aim of the study. Substantial improvements in accuracy are observed although further enhancements are needed before metamodels can capture all kinds of responses accurately. 


\section{Paper V}

\section{Improving accuracy of feedforward neural network metamodels for crashworthiness optimization}

In this paper, the approaches found most promising in Paper IV are refined and further studied. Again, it is found that both SVM-based sequential sampling and augmenting the metamodel fitting set with estimated guiding samples can improve the accuracy of feedforward neural network metamodels. In addition, it is concluded that an architecture with two hidden layers, instead of one, often gives lower error measures for the tested responses. Further, the presented examples, including an industrial application, prove that the increased accuracy offered by the proposed methods can result in successful optimization studies for cases where more conventional methods fail. 
CHAPTER 6. SUMMARY OF APPENDED PAPERS 


\section{Conclusions and Outlook}

The primary goal of this research has been to find suitable methods for large-scale multidisciplinary design optimization of automotive structures that can be implemented within current product development. The assessment of different MDO methods in Paper I shows that a single-level method in combination with global metamodels is generally the most suitable approach. The metamodel-based MDO process in six steps presented in Paper II fits into the organization of automotive companies since existing groups of disciplinary experts can work concurrently and autonomously on the most time-consuming steps of the process. It also fits a CAE-based development process where validated simulation models of the same status are available for all load cases at the virtual assessments.

The presented process can be applied to different types of MDO studies and it is proven efficient in Paper II for a thickness optimization study with the objective to minimize mass. The number of design variables in such size optimization studies is normally limited. When the number of spot welds in a structure should be determined, the number of variables is often much larger. By parameterizing the model, as in Paper III, and let the design variables represent the number of spot welds in an area, the proposed MDO process can be used also for this type of problem. Alternatively, topology optimization that requires less model preparation and computational effort can be used. However, topology optimization methods are generally not truly multidisciplinary and therefore only suitable for studies where load cases involving simulations of linear systems are judged to be of major importance.

Even though it is concluded in Paper II that useful results can be obtained by employing the proposed MDO process with metamodels of limited accuracy, the chances of successful outcomes increase if metamodels with small prediction errors are used. Complex responses including discontinuities that are common in for example impact situations are especially hard to capture by global metamodels. The idea to use the decision boundary from a support vector machine to discriminate between different types of deformation behaviour in the design space and thereby improve metamodel accuracy is elaborated upon in Paper IV and V. Three different approaches are tested; to split the design space and fit separate metamodels for the different regions, to augment the fitting set with estimated guiding samples along the boundary before a global metamodel is fitted, and to use a special SVM-based sequential sampling method. It is concluded that the split design 
space approach is only suitable for responses with a step-like behaviour. The estimated guiding samples approach is more general and can improve the accuracy for discontinuous responses without decreasing it for smooth ones. The results from the studied examples show that the SVM-based sequential sampling technique generally results in lower error measures for the feedforward neural network metamodels than more conventional spacefilling DOEs. Lowest error measures are obtained for metamodels developed either with or without estimated guiding samples. It is also found that it is often beneficial to use two hidden layers instead of one for complex responses. Since it is often unknown which approach that will give the best results, it is recommended that several sets of metamodels are developed. Performing MBDO with different sets of metamodels offers a way to obtain additional design proposals at a small extra effort, which can turn out to be valuable. In Paper V, it is shown that the proposed methods can result in successful optimization studies for cases where more conventional methods fail.

Although the methods in this thesis are developed with large-scale applications in mind, some of them are verified in problems of limited size. By necessity, the number of test cases is also restricted. Additional testing on large industrial applications will clarify the limitations of the proposed methods and reveal what further improvements are needed. Such improvements can include improved SVM-based sequential sampling where the balance between exploiting and exploring samples are automatically determined and can vary between steps. Further, the appropriate number of estimated guiding samples can be investigated in more detail. However, already now the suggested MDO process combined with the presented measures to improve metamodel accuracy has the potential to efficiently improve and balance product performance. For companies that already follow a CAE-based development process, the implementation should be straightforward and largescale multidisciplinary design optimization of automotive structures can be made an efficient part of the daily work. 


\section{References}

Backlund P. B., Shahan D. W., Seepersad C. C. (2012) A comparative study of the scalability of alternative metamodelling techniques. Engineering Optimization, 44(7), 767-786.

doi:10.1080/0305215X.2011.607817

Basudhar, A. (2011). Computational optimal design and uncertainty quantification of complex systems using explicit decision boundaries. Ph.D. thesis, UMI No. 3464753. Department of Aerospace and Mechanical Engineering, The University of Arizona.

Bendsøe, M. P. (1989) Optimal shape design as a material distribution problem. Structural Optimization 1(4), 193-202.

doi:10.1007/BF01650949

Ben-Hur, A., Weston, J. (2010) A user's guide to support vector machines. In: Carugo, O., Eisenhaber, F. (Eds.), Data Mining Techniques for the Life Sciences (pp. 223-239). Totowa, NJ: Humana Press. doi:10.1007/978-1-60327-241-4_13

Beyer, H.-G., Schwefel, H.-P. (2002). Evolution strategies: A comprehensive introduction. Natural Computing, 1(1), 3-52. doi:10.1023/A:1015059928466.

Boser, B. E., Guyon, I. M., Vapnik, V. N. (1992) A training algorithm for optimal margin classifiers. Fifth Annual Workshop on Computational Learning Theory. Pittsburgh, PA, July 27-29 (pp. 144-152). doi:10.1145/130385.130401

Braun, R. D. (1996). Collaborative optimization: An architecture for large-scale distributed design. Ph.D. thesis, UMI No. 9630279. Department of Aeronautics and Astronautics, Stanford University.

Clarke, S. M., Griebsch, J. H., Simpson, T. W. (2005). Analysis of support vector regression for approximation of complex engineering analyses. Journal of Mechanical Design, 127(6), 1077-1087.

doi:10.1115/1.1897403 
Craig, K. J., Stander, N., Dooge, D. A., Varadappa, S. (2002). MDO of automotive vehicle for crashworthiness and NVH using response surface methods (AIAA 2002-5607). 9th AIAA/ISSMO Symposium on Multidisciplinary Analysis and Optimization. Atlanta, GA, Sep. 4-6 (pp. 1930-1940).

doi: $10.2514 / 6.2002-5607$

Cortes, C., Vapnik, V. (1995) Support-vector networks. Machine Learning, 20(3), 273-297.

doi:10.1007/BF00994018

Cramer, E. J., Dennis Jr., J. E., Frank, P. D., Lewis, R. M., Shubin, G. R. (1994). Problem formulation for multidisciplinary optimization. SIAM Journal on Optimization, 4(4), 754-776.

doi: $10.1137 / 0804044$

Duddeck, F. (2008). Multidisciplinary optimization of car bodies. Structural and Multidisciplinary Optimization, 35(4), 375-389.

doi:10.1007/s00158-007-0130-6

Friedman, J. H. (1991). Multivariate adaptive regression splines. The Annals of Statistics, 19(1), 1-67. doi: $10.2307 / 2241837$

Giesing, J. P., Barthelemy, J. M. (1998). A summary of industry MDO applications and needs (AIAA 1998-4737). 7th AIAA/USAF/NASA/ISSMO Symposium on Multidisciplinary Analysis and Optimization. St. Louis, MO, Sep. 2-4. doi:10.2514/6.1998-4737

Goel, T., Stander, N. (2009). Comparing three error criteria for selecting radial basis function network topology. Computer Methods in Applied Mechanics and Engineering, 198(27-29), 2137-2150.

doi:10.1016/j.cma.2009.02.016

Gunn, S. R. (1998) Support vector machines for classification and regression. Technical report ISIS-1-98. School of Electronics and Computer Science, University of Southampton.

Han, J., Papalambros, P. Y. (2010) Optimal design of hybrid electric fuel cell vehicle under uncertainty and enterprise considerations. Journal of Fuel Cell Science and Technology, 7(2), 021020-1-9.

doi:10.1115/1.3179762

Hardy, R. L. (1990). Theory and applications of the multiquadric-biharmonic method: 20 years of discovery 1968-1988. Computers \& Mathematics with Applications, 19(8-9), 163-208. doi:10.1016/0898-1221(90)90272-L 
Holland, J. H. (1992). Adaptation in natural and artificial systems: An introductory analysis with applications to biology, control, and artificial intelligence. Cambridge, MA: MIT Press.

Hoppe, A., Kaufmann, M., Lauber, B. (2005). Multidisciplinary optimization considering crash and NVH loadcases. ATZ/MTZ Virtual Product Creation. Stuttgart, June 20-21.

Hornik, K., Stinchcombe, M., White, H. (1989). Multilayer feedforward networks are universal approximators. Neural Networks, 2(5), 359-366.

doi:10.1016/0893-6080(89)90020-8

Ingber, L. (1996). Adaptive simulated annealing (ASA): Lessons learned. Control and Cybernetics, 25(1), 33-54.

Iooss, B., Boussouf, L., Feuillard, V., Marrel, A. (2010). Numerical studies of the metamodel fitting and validation processes. International Journal on Advances in Systems and Measurements, 3(1-2), 11-21.

Jin, R., Chen, W., Simpson, T. (2001). Comparative studies of metamodelling techniques under multiple modelling criteria. Structural and Multidisciplinary Optimization, 23(1), 1-13. doi:10.1007/s00158-001-0160-4

Jin, R., Chen, W., Sudjianto, A. (2002). On sequential sampling for global metamodeling in engineering design (DETC2002/DAC-34092). ASME 2002 International Design Engineering Technical Conferences and Computers and Information in Engineering Conference. Montreal, Sep. 29-Oct. 2 (pp. 539-548).

doi:10.1115/DETC2002/DAC-34092

Johnson, M., Moore, L., Ylvisaker, D. (1990). Minimax and maximin distance designs. Journal of Statistical Planning and Inference, 26(2), 131-148. doi:10.1016/0378-3758(90)90122-B

Kang, N., Kokkolaras, M., Papalambros, P. Y., Yoo, S., Na, W., Park, J., Featherman, D. (2014) Optimal design of commercial vehicle systems using analytical target cascading. Structural and Multidisciplinary Optimization, 50(6), 1103-1114. doi:10.1007/s00158-014-1097-8

Kim, B.-S., Lee, Y.-B., Choi, D.-H. (2009). Comparison study on the accuracy of metamodeling technique for non-convex functions. Journal of Mechanical Science and Technology, 23(4), 1175-1181. doi:10.1007/s12206-008-1201-3

Kim, H. M. (2001). Target cascading in optimal system design. Ph.D. thesis, UMI No. 3016884. Department of Mechanical Engineering, The University of Michigan. 
Kim, H. M., Michelena, N. F., Papalambros, P. Y., Jiang, T. (2003). Target cascading in optimal system design. Journal of Mechanical Design, 125(3), 474-480. doi:10.1115/1.1582501

Kim, H. M., Kokkolaras, M., Louca, L. S., Delagrammatikas, G. J., Michelena, N. F., Filipi, Z. S., Papalambros, P. Y., Stein, J. L., Assanis, D. N. (2002). Target cascading in vehicle redesign: A class VI truck study. International Journal of Vehicle Design, 29(3), 199-225. doi:10.1504/IJVD.2002.002010

Kirkpatrick, S., Gelatt, C. D., Vecchi, M. P. (1983). Optimization by simulated annealing. Science, 220(4598), 671-680. doi: $10.1126 /$ science.220.4598.671

Kodiyalam, S., Sobieszczanski-Sobieski, J. (2001). Multidisciplinary design optimization - Some formal methods, framework requirements, and application to vehicle design. International Journal of Vehicle Design, 25(1-2), 3-22. doi:10.1504/IJVD.2001.001904

Kodiyalam, S., Yang, R., Gu, L., Tho, C.-H. (2004). Multidisciplinary design optimization of a vehicle system in a scalable, high performance computing environment. Structural and Multidisciplinary Optimization, 26(3), 256-263. doi:10.1007/s00158-003-0343-2

Kroo, I., Altus, S., Braun, R., Gage, P., Sobieski, I. (1994). Multidisciplinary optimization methods for aircraft preliminary design (AIAA-94-4325). 5th AIAA/USAF/NASA/ISSMO Symposium on Multidisciplinary Analysis and Optimization. Panama City Beach, FL, Sep. 7-9 (pp. 697-707). doi:10.2514/6.1994-4325

Li, Y. F., Ng, S. H., Xie, M., Goh, T. N. (2010). A systematic comparison of metamodeling techniques for simulation optimization in decision support systems. Applied Soft Computing, 10(4), 1257-1273. doi:10.1016/j.asoc.2009.11.034

Martin, J. D., Simpson, T. W. (2004). On the use of Kriging models to approximate deterministic computer models (DETC2004/DAC-57300). ASME 2004 International Design Engineering Technical Conferences and Computers and Information in Engineering Conference. Salt Lake City, UT, Sep. 28-Oct. 2 (pp. 481-492). doi:10.1115/DETC2004-57300

Martins, J. R. R. A., Lambe, A. B. (2013). Multidisciplinary design optimization: A survey of architectures. AIAA Journal, 51(9), 2049-2075. doi:10.2514/1.J051895 
McKay, M. D., Beckman, R. J., Conover, W. J. (1979) A comparison of three methods for selecting values of input variables in the analysis of output from a computer code. Technometrics 21(2), 239-245.

doi:10.1080/00401706.1979.10489755

Michalek, J. J., Papalambros, P. Y. (2005). Weights, norms, and notation in analytical target cascading. Journal of Mechanical Design, 127(3), 499-501. doi:10.1115/1.1862674

Myers, R. H., Montgomery, D. C., Andersson-Cook, C. M. (2009). Response surface methodology: Process and product optimization using designed experiments ( $3^{\text {rd }}$ ed.). Hoboken, NJ: Wiley.

Patel, N. M., Kang, B.-S., Renaud, J. E., Tovar, A. (2009) Crashworthiness design using topology optimization. Journal of Mechanical Design 131(6), 061013-1-12. doi: $10.1115 / 1.3116256$

Queipo, N. V., Haftka, R. T., Shyy, W., Goel, T., Vaidyanathan, R., Tucker, P. K. (2005). Surrogate-based analysis and optimization. Progress in Aerospace Sciences, 41(1), $1-28$.

doi:10.1016/j.paerosci.2005.02.001

Rakowska, J., Chator, A., Barthelemy, B., Lee, M., Morgans, S., Laya, J., Zinn, G., Chuang, C.-H., Gondipalle, S. R. (2015). An iterative application of multidisciplinary optimization for vehicle body weight reduction based on 2015 Mustang product development. SAE International Journal of Materials and Manufacturing, 8(3), 685-692.

doi: $10.4271 / 2015-01-0470$

Roth, B. D. (2008). Aircraft family design using enhanced collaborative optimization. Ph.D. thesis, UMI No. 3332916. Department of Aeronautics and Astronautics, Stanford University.

Ryberg, A.-B. (2013). Metamodel-based design optimization - A multidisciplinary approach for automotive structures. Licentiate thesis No. 1565. Division of Solid Mechanics, Linköping University.

Sacks, J., Welch, W. J., Mitchell, T. J., Wynn, H. P. (1989). Design and analysis of computer experiments. Statistical Science, 4(4), 409-423. doi: $10.2307 / 2245858$

Sheldon, A., Helwig, E., Cho, Y.-B. (2011). Investigation and application of multidisciplinary optimization for automotive body-in-white development. 8th European LS-DYNA Users Conference. Strasbourg, May 23-24. 
Shetty, S. (2017) Optimization of vehicle structures under uncertainties. Ph.D. thesis No. 1809. Division of Solid Mechanics, Linköping University. doi:10.3384/diss/diva-133199

Smola, A. J., Schölkopf, B. (2004). A tutorial on support vector regression. Statistics and Computing, 14(3), 199-222. doi:10.1023/B:STCO.0000035301.49549.88

Sobieszczanski-Sobieski, J. (1988). Optimization by decomposition: A step from hierarchic to non-hierarchic systems. Second NASA/Air Force Symposium on Recent Advances in Multidisciplinary Analysis and Optimization. Hampton, VA, Sep. 28-30.

Sobieszczanski-Sobieski, J., Agte, J. S., Sandusky Jr., R. R. (1998). Bi-level integrated system synthesis (BLISS) (AIAA-98-4916). 7th AIAA/USAF/NASA/ISSMO Symposium on Multidisciplinary Analysis and Optimization. St. Louis, MO, Sep 2-4 (pp. 1543-1557). doi:10.2514/6.1998-4916

Sobieszczanski-Sobieski, J., Kodiyalam, S., Yang, R. (2001). Optimization of car body under constraints of noise, vibration, and harshness (NVH), and crash. Structural and Multidisciplinary Optimization, 22(4), 295-306.

doi:10.1007/s00158-001-0150-6

Sobol', I. M. (2001). Global sensitivity indices for nonlinear mathematical models and their Monte Carlo estimates. Mathematics and Computers in Simulation, 55(1-3), 271-280.

doi:10.1016/S0378-4754(00)00270-6

Stander, N., Roux, W., Basudhar, A., Eggleston, T., Goel, T., Craig, K. (2015). LS-OPT® user's manual, Version 5.2. Livermore: Livermore Software Technology Corporation.

Tosserams, S., Etman, L. F. P., Papalambros, P. Y., Rooda, J. E. (2006). An augmented Lagrangian relaxation for analytical target cascading using the alternating direction method of multipliers. Structural and Multidisciplinary Optimization, 31(3), 176189.

doi:10.1007/s00158-005-0579-0

Viana, F. A., Gogu, C., Haftka, R. T. (2010). Making the most out of surrogate models: tricks of the trade (DETC2010-28813). ASME 2010 International Design Engineering Technical Conferences and Computers and Information in Engineering Conference, Montreal, Aug. 15-18 (pp. 587-598).

doi:10.1115/DETC2010-28813 
Viana, F. A. C., Venter, G., Balabanov, V. (2010) An algorithm for fast optimal Latin hypercube design of experiments. International Journal for Numerical Methods in Engineering, 82(2), 135-156.

doi:10.1002/nme. 2750

Xue, Z., Elango, A., Fang, J. (2016). Multidisciplinary design optimization of vehicle weight reduction. SAE International Journal of Materials and Manufacturing, 9(2), 393-399.

doi:10.4271/2016-01-0301

Yang, R. J., Gu, L., Tho, C. H., Sobieszczanski-Sobieski, J. (2001). Multidisciplinary design optimization of a full vehicle with high performance computing (AIAA2001-1273). 42nd AIAA/ASME/ASCE/AHS/ASC Structures, Structural Dynamics, and Materials Conference and Exhibit. Seattle, WA, Apr 16-19.

doi:10.2514/6.2001-1273 


\section{Papers}

The papers associated with this thesis have been removed for copyright reasons. For more details about these see:

http://urn.kb.se/resolve?urn=urn:nbn:se:liu:diva-140876 